(C) Copyright 2012 American Meteorological Society (AMS). Permission to use figures, tables, and brief excerpts from this work in scientific and educational works is hereby granted provided that the source is acknowledged. Any use of material in this work that is determined to be "fair use" under Section 107 of the U.S. Copyright Act or that satisfies the conditions specified in Section 108 of the U.S. Copyright Act (17 USC §108, as revised by P.L. 94-553) does not require the AMS's permission. Republication, systematic reproduction, posting in electronic form, such as on a web site or in a searchable database, or other uses of this material, except as exempted by the above statement, requires written permission or a license from the AMS. Additional details are provided in the AMS Copyright Policy, available on the AMS Web site located at (http://www.ametsoc.org/) or from the AMS at 617-227-2425 or copyright@ametsoc.org. 


\title{
Quantification of Cloud Microphysical Parameterization Uncertainty Using Radar Reflectivity
}

\author{
MARCUS VAN LIER-WALQUI \\ Rosenstiel School of Marine and Atmospheric Science, University of Miami, Miami, Florida \\ TOMISLAVA VUKICEVIC \\ Hurricane Research Division, NOAA/AOML, Miami, Florida \\ DEREK J. POSSELT \\ University of Michigan, Ann Arbor, Ann Arbor, Michigan
}

(Manuscript received 15 August 2011, in final form 15 May 2012)

\begin{abstract}
Uncertainty in cloud microphysical parameterization - a leading order contribution to numerical weather prediction error-is estimated using a Markov chain Monte Carlo (MCMC) algorithm. An inversion is performed on 10 microphysical parameters using radar reflectivity observations with vertically covarying error as the likelihood constraint. An idealized 1D atmospheric column model with prescribed forcing is used to simulate the microphysical behavior of a midlatitude squall line. Novel diagnostics are employed for the probabilistic investigation of individual microphysical process behavior vis-à-vis parameter uncertainty. Uncertainty in the microphysical parameterization is presented via posterior probability density functions (PDFs) of parameters, observations, and microphysical processes. The results of this study show that radar reflectivity observations, as expected, provide a much stronger constraint on microphysical parameters than column-integral observations, in most cases reducing both the variance and bias in the maximum likelihood estimate of parameter values. This highlights the enhanced potential of radar reflectivity observations to provide information about microphysical processes within convective storm systems despite the presence of strongly nonlinear relationships within the microphysics model. The probabilistic analysis of parameterization uncertainty in terms of both parameter and process activity PDFs suggest the prospect of a stochastic representation of microphysical parameterization uncertainty—specifically the results indicate that error may be more easily represented and estimated by microphysical process uncertainty rather than microphysical parameter uncertainty. In addition, these new methods of analysis allow for a detailed investigation of the full nonlinear and multivariate relationships between microphysical parameters, microphysical processes, and radar observations.
\end{abstract}

\section{Introduction}

Accurate prediction of precipitation is one of the greatest challenges of numerical weather prediction (NWP). The physical processes controlling precipitation occur at a microscopic scale and thus cannot be explicitly represented at the model resolution. The parameterization of cloud and precipitation microphysics is required, which estimates the contribution of the subgrid-scale

Corresponding author address: Marcus van Lier-Walqui, RSMAS, University of Miami, 4600 Rickenbacker Cswy., Miami, FL 33130.

E-mail: mvanlier-walqui@rsmas.miami.edu processes on the model grid. The cloud and precipitation microphysical parameterizations include a set of prespecified parameters that approximate physical properties of some assumed distribution of different classes of hydrometeors. This representation is necessarily characterized by uncertainty or error. Quantification of the parameterization uncertainty is crucial for the purposes of improving microphysical parameterization; in particular, it is important to understand how observations might constrain parameterization uncertainty. This knowledge can then be used to inform attempts to improve the fidelity of microphysical parameterization schemes through the assimilation of observations. In this work, the formalism of probabilistic inverse modeling is 
chosen in order to characterize microphysical parameterization uncertainty (Mosegaard and Tarantola 2002; Tarantola 2005; Vukicevic and Posselt 2008, hereafter VP08). The current study can thus be thought of as a convolution of the information spaces of simulated observations and a choice of model control parameters. The set of model microphysical parameters and observations of the modeled storm are treated as probabilistic measures with associated probability density functions (PDFs). These PDFs represent the likelihood of possible, realistic parameter values given observational information. This approach is general and allows, in particular, for the exploration of interdependent parameter uncertainty as well as uncertainty that is not Gaussian or unimodal. Additionally, the probabilistic framework employed is used to present uncertainty in the microphysical modeling system in terms of posterior PDFs of microphysical processes, microphysical parameters, radar reflectivity observations, and the error dependence between these variables.

The focus of the current study is on uncertainty within a single-moment bulk microphysical parameterization scheme, specifically a modified version of Lin et al. (1983) (Tao et al. 1989; Tao and Simpson 1993; Tao et al. 2003; Posselt 2006; Lang et al. 2007; Posselt and Vukicevic 2010, hereafter PV10). The technique employed here, however, is generally applicable to any control parameters of any model. Many studies have investigated the effect of microphysical parameterization choices on cloud-resolving model (CRM) behavior, however, most are limited to the consideration of relatively few variations in parameterization. One such approach has been to compare the behavior of disparate schemes within idealized frameworks (Stensrud et al. 2000; Thompson et al. 2004; Morrison and Grabowski 2007; Morrison et al. 2009; Li et al. 2009) or real cases (McCumber et al. 1991; Jankov et al. 2007; Clark et al. 2008; Gallus and Pfeifer 2008). Another approach has been to perform sensitivity tests of a single parameter within a single microphysics scheme (Thompson et al. 2004; Milbrandt and Yau 2005). These studies lend valuable insight into differences in storm morphology with perturbed model microphysics, however, they are limited in their exploration of the full breadth of parameter variability. That is to say, the former type compares entirely different choices of control parameters (and then chooses a single value for these parameters); whereas the latter typically varies only a single parameter at a time (in essence, tracing a onedimensional line within a multidimensional parameter space). These concessions are made so that the CRM can be run in something like an operational or real-time mode, with full thermodynamic and dynamic feedback. There are few examples of studies which have explored model sensitivity to simultaneous perturbation of parameters; typically this has been attempted for land surface model parameters using regional sensitivity analysis, a method that uses Monte Carlo sampling and the Kolmogorov-Smirnov probability to test for influential parameters (Liu et al. 2004; Ott et al. 2009). These tests, however, are not capable of diagnosing nonlinear or multimodal relationships between parameters and model state.

The current study has more in common with work in parameter estimation, especially studies using Monte Carlo techniques that sample from a generally nonGaussian parameter posterior distribution, such as veryfast simulated annealing (VFSA) used in land surface and climate model parameters (Jackson et al. (2003, 2008), respectively) or adaptive Markov chain Monte Carlo (MCMC), used again for climate parameter estimation (Järvinen et al. 2010). Other ensemble parameter estimation techniques, including an innovative online framework (Järvinen et al. 2012) and a technique by which ensemble members are weighted by their likelihood (Golaz et al. 2007) also aim to estimate the PDF of parameters, however, with many fewer Monte Carlo members. Besides PV10, the authors know of no study that has attempted to estimate the full nonlinear, nonGaussian uncertainty of parameters within a microphysics scheme, or how that uncertainty affects individual modeled processes. Furthermore, the MCMC technique used here typically provides a more accurate sample of the posterior parameter PDF than VFSA (Sen and Stoffa 1996; Jackson et al. 2003) or techniques with limited ensemble size.

The current work is not a traditional sensitivity study, nor is it an optimization; like the parameter estimation studies just mentioned, the end result is a full multivariate posterior PDF representing parameterization uncertainty conditioned on observations - uncertainty that may be nonlinear and multimodal. The probabilistic approach employed additionally allows for novel diagnostics of the individual microphysical processes modeled in the parameterization scheme, such that the posterior uncertainty may be represented in terms of these processes as well as in terms of parameter values. The result takes the form of a fully multivariate posterior joint parameter-process PDF; in general, the relationships shown between each parameter and modeled process will be different from the deterministic relationships specified by the governing equations due to the fact that model state and all parameters of interest may vary simultaneously.

This study follows PV10 in which the same 1D CRM is used to estimate uncertainty of 10 physical parameters, constrained by observations of column-integral 
TABLE 1. Cloud microphysical parameter descriptions, abbreviation, along with truth values and parameter ranges. Table reproduced from PV10.

\begin{tabular}{|c|c|c|c|c|c|}
\hline Parameter description & Abbreviation & Units & Truth & Min & Max \\
\hline Snowfall speed coefficient & $a_{s}$ & $\mathrm{~cm}^{1-b_{s}}$ & 200.0 & 50.0 & 1000.0 \\
\hline Snowfall speed exponent & $b_{s}$ & none & 0.3 & 0.1 & 1.0 \\
\hline Graupel fall speed coefficient & $a_{g}$ & $\mathrm{~cm}^{1-b_{g}}$ & 400.0 & 50.0 & 1200.0 \\
\hline Graupel fall speed exponent & $b_{g}$ & none & 0.4 & 0.1 & 0.9 \\
\hline Intercept parameter, rain particle size distribution & $N_{0 r}$ & $\mathrm{~cm}^{-4}$ & 0.5 & 0.0 & 5.0 \\
\hline Intercept parameter, snow particle size distribution & $N_{0 \mathrm{~s}}$ & $\mathrm{~cm}^{-4}$ & 0.5 & 0.0 & 5.0 \\
\hline Intercept parameter, graupel particle size distribution & $N_{0 g}$ & $\mathrm{~cm}^{-4}$ & 0.5 & 0.0 & 5.0 \\
\hline Snow particle density & $\rho_{s}$ & $\mathrm{~g} \mathrm{~cm}^{-3}$ & 0.2 & 0.1 & 1.0 \\
\hline Graupel particle density & $\rho_{g}$ & $\mathrm{~g} \mathrm{~cm}^{-3}$ & 0.4 & 0.1 & 1.0 \\
\hline Threshold cloud mixing ratio for autoconversion to rain & $q_{\mathrm{c} 0}$ & $\mathrm{~g} \mathrm{~kg}^{-1}$ & 1.0 & 0.1 & 3.0 \\
\hline
\end{tabular}

quantities of precipitation rate, liquid and ice water path, and outgoing shortwave and outgoing longwave radiation. In the current study, radar reflectivity is chosen as the observable quantity because of its ubiquity as a tool for evaluating precipitating weather features. Radar reflectivity measurements are generally vertically resolved, which allows for an estimation of the vertical structure of storms. In addition, radar reflectivity is sensitive to the phase and particle size distribution of hydrometeors and is thus capable of providing information for the inversion of microphysical parameters. Comparison of the current results with PV10 allows for an objective estimation of the additional information provided by the vertically resolved radar reflectivity observations.

\section{Experimental design}

The experimental system here is based on work described in PV10, where a more detailed overview can be found. The method will thus only be described briefly. The model consists of a one-dimensional atmospheric column with a bulk microphysics parameterization (Lin et al. 1983; Tao et al. 1989; Posselt 2006). The atmospheric column is forced with prescribed time-varying profiles of water vapor, temperature, and vertical velocity in order to simulate the variety of microphysical processes that occur within a prototypical midlatitude squall line. Storm behavior along the time dimension varies in a manner analogous to behavior along the horizontal dimension in two-dimensional CRM studies of squall lines (Li et al. 2009; Morrison and Grabowski 2007), and as such goes through a "convective" morphological phase followed by a "stratiform" phase (PV10). The column model is not intended to simulate real weather, but rather to serve as a driver for a diversity of microphysical processes present within a prototypical modeled squall line. The model is thus a useful offline tool for the investigation of modeled microphysical behavior, in the spirit of recent work by Shipway and Hill (2012) and Dearden et al. (2010). The time-varying forcing profiles and the hydrometeor concentrations produced by the model under these forcings are shown in Fig. 1 and 2 of PV10, respectively.

A total of 10 microphysical parameters are chosen for their importance within the equations describing microphysical processes; these parameters are listed in Table 1. For each parameter, a "truth" value is defined as well as minimum and maximum realistic values (which form the prior parameter information used in the inverse experiment). When the model is integrated using the truth parameters, the results are considered the synthetic true state of the atmosphere, and simulated observations of this model state are deemed observational truth (this is represented in gray in the schematic shown in Fig. 2). As with real observations, the simulated observation is stochastic, and can be defined by a PDF. Assuming a Gaussian distribution for the observational uncertainty, this PDF is defined by two quantities-the mean, or first moment, and the covariance, or second moment. The observational truth is used to define the mean, implying unbiased observations. The covariance can be estimated using some assumptions of observational uncertainty that will be discussed later in this section.

The choice of the microphysical parameters of interest defines a 10-dimensional control parameter space. This space is explored so as to determine the 10-dimensional probability density function of the parameters conditioned on information in the observations. To achieve this, an MCMC algorithm is employed. MCMCs define a class of statistical techniques used to sample a posterior probability distribution, returning an estimate of that PDF in an efficient, robust manner. The reader is referred to PV10 as well as Robert and Casella (2004) and Posselt et al. (2008) for further details about MCMC algorithms. The MCMC algorithm employed uses the Metropolis sampler (Metropolis et al. 1953), which will be briefly described here. 
A schematic for the Metropolis sampler used is shown in blue (in Fig. 2). The sampler starts from some random initial point within the 10-dimensional control parameter state. The forward model is integrated and simulated observations of radar reflectivity are generated at the desired observation times. A cost function is used to calculate the misfit between these forward observations and the truth observations generated with the truth parameters. For the assumed Gaussian error distribution in the observation space, the cost function is a loglikelihood function (Tarantola 2005), which includes covariance between errors at different levels within the radar profile. The cost function could also include temporal error correlation when using measurements from different time instances or equivalently from different sections of the corresponding composite squall-line cross section; however, temporal observation error correlations are neglected in the current study. The likelihood is thus $e^{-\boldsymbol{\Phi}_{\mathbf{x}, \mathbf{y}}}$, where $\boldsymbol{\Phi}_{\mathbf{x}, \mathbf{y}}$ is the cost function. The cost function for parameters $\mathbf{x}$ given observations $\mathbf{y}$, forward model $F$, and observation error covariance matrix $\mathbf{R}$ is given by

$$
\boldsymbol{\Phi}_{\mathbf{x}, \mathbf{y}}=\frac{1}{2}[\mathbf{y}-F(\mathbf{x})]^{\mathrm{T}} \mathbf{R}^{-1}[\mathbf{y}-F(\mathbf{x})] .
$$

The sampler then simultaneously performs a random step in all 10 dimensions, with the resultant parameter set deemed the proposal. The forward model is integrated and simulated observations are produced that can be used to calculate the cost. The parameter proposal is accepted with a probability of $\min \left(1, e^{\boldsymbol{\Phi}_{\text {prior }}-\boldsymbol{\Phi}_{\text {proposal }}}\right)$ and thus proposals with greater likelihood than the prior are always accepted, whereas proposals with a lower likelihood are allowed with a probability given by the ratio of proposal and prior likelihoods. Note that the full posterior probability being sampled also includes a prior distribution on the parameters, which in this case is a bounded uniform distribution. This choice is made so as to be close to noninformative while still forbidding unrealistic ranges of the parameter values (PV10). To include this prior parameter probability, any proposals outside the parameter bounds (specified in Table 1) are treated as having probability $=0$ and are rejected. In the case of a rejected proposal, the prior parameter set is accepted into the chain rather than the proposal.

Radar reflectivity simulated observations are generated using the Quickbeam radar forward operator (Haynes et al. 2007) for a 3-GHz radar frequency [cf. Weather Surveillance Radar-1988 Doppler (WSR-88D) radar frequency]. This operator is capable of solving Mie equations for a variety of radar frequencies and a variety of user-specified particle size distributions and microphysical parameter values. In contrast to the

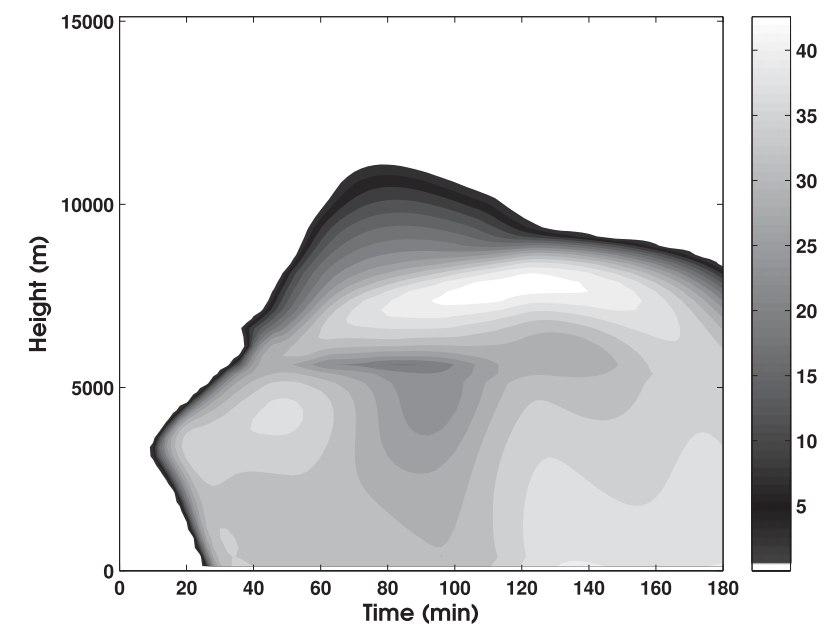

FIG. 1. Radar reflectivity (dBZ) for "truth" model run. Calculated using Quickbeam radar reflectivity operator (Haynes et al. 2007).

work of Tong and Xue (2008), the perturbed values of the microphysical parameters are used in the radar forward operator. In this sense, the CRM and the radar forward operator can be thought of as constituting a single, consistent forward model. In tests (not shown here), Quickbeam was found to be comparable to other available forward operators such as the Goddard Satellite Data Simulation Unit (G-SDSU) (Masunaga et al. 2010) in relative distribution of reflectivity, with the exception of the melting layer reflectivity bright band, which Quickbeam is unable to reproduce. Radar reflectivity is simulated at model grid resolution and beam bending, broadening, and attenuation are not considered because of the idealized nature of this investigation (N.B. in inversions using real observations, these and other unaccounted-for sources of error may be important). Simulated radar reflectivity using the reference truth values of the parameters in the model is shown in Fig. 1. From this solution measurements are selected from two distinct storm morphological regimes: convective at $60 \mathrm{~min}$ and stratiform at $120 \mathrm{~min}$.

The cost function used must take into account observational errors as well as errors in the radar model. This error is vertically correlated and its covariance matrix is evaluated from a sample of atmospheric states that represent uncertainty in the modeled atmosphere. This representative sample of atmospheric profiles is generated by a MCMC experiment that reflects prior knowledge of the atmosphere. The ensemble of the parameter estimates from PV10 using five column-integrated measures as observational constraint is used to define this prior knowledge. From these data the ensemble of radar reflectivity simulated observations is computed from which a matrix of vertical covariance in simulated radar 


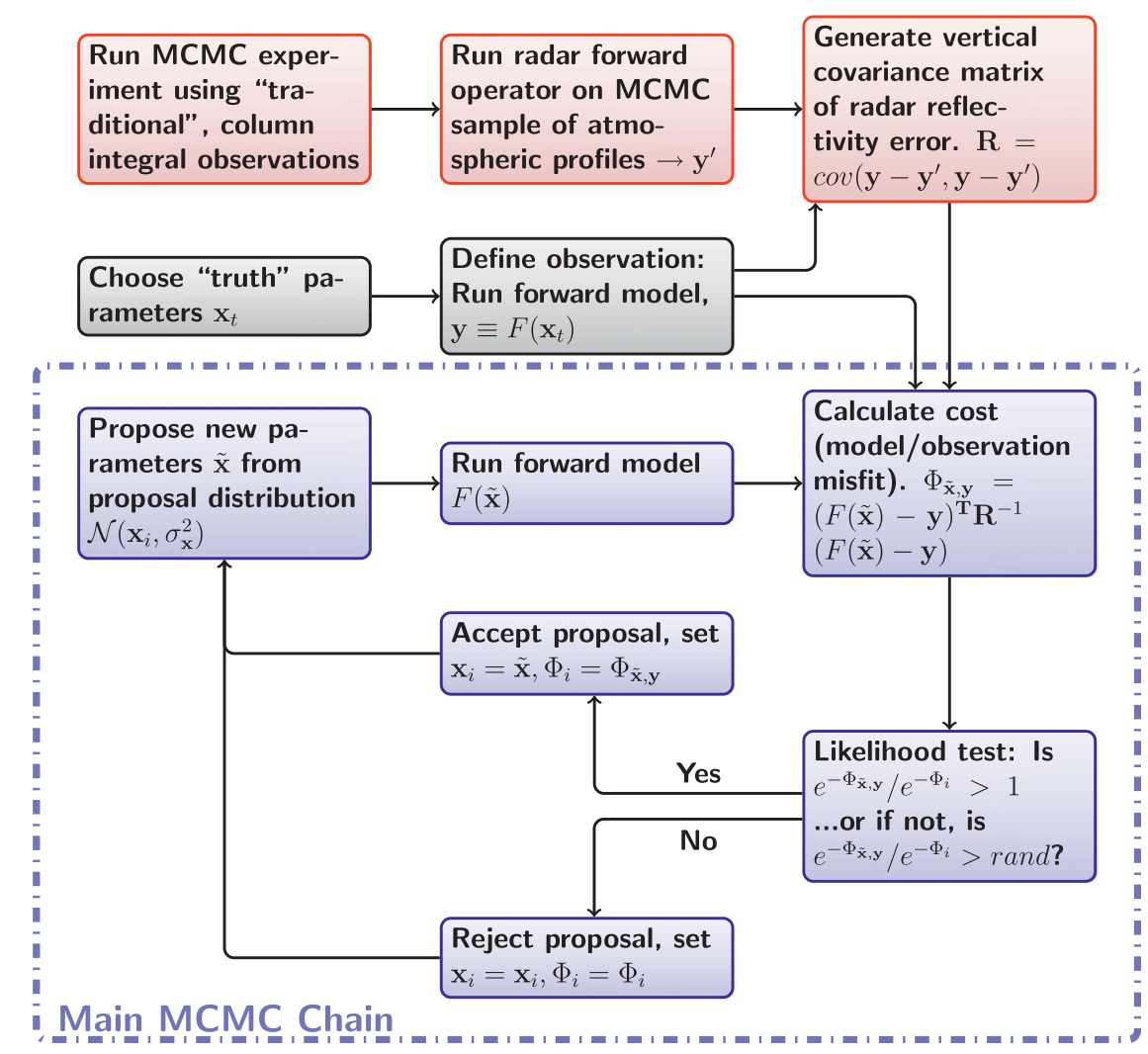

FIG. 2. Schematic of MCMC algorithm with radar reflectivity error covariance estimation (red), truth observation generation (gray), and main MCMC loop (blue).

reflectivity error is generated for each model output time step. The variance is then inflated by a factor of 1.3 in order to facilitate matrix inversion as well as to provide consideration of errors not addressed by the aforementioned model. This particular value of the variance inflation factor was chosen ad hoc due to its ability to improve MCMC sampler performance without strongly altering values of observational error. A schematic for the generation of the observational error covariance matrix is shown in red in Fig. 2.

\section{Results}

The Markov chain represents the probability density function of all model variables; in this case only microphysical parameters were directly perturbed, however, all model variables and simulated observations affected by these perturbations may be described by a probability density function under the constraints of the chosen model-observational system. The 10-dimensional parameter PDFs are presented here in addition to joint PDFs for parameters and observations, parameters and microphysical process activity, and microphysical process activity PDFs. First, the radar reflectivity error covariance is presented, which highlights correlations in the vertical structure of simulated reflectivity error and forms this study's assumptions about vertically covarying observational error. Next, the 10-dimensional posterior microphysical parameter PDF is shown. These data represent the solution to the inverse problem and are robust estimates of uncertainty in the parameters when constrained by the radar reflectivity observations. Joint posterior PDFs are then shown, which combine variable types to show joint sensitivity and multivariate, nonlinear relationships of uncertainty. First, parameterobservation joint posterior PDFs are presented, which show sensitivity of simulated observations to simultaneous parameter perturbation as well as specific information about how observational data provide constraint on parameters. Then parameter-process joint posterior PDFs are presented, which show the modeled process sensitivity to parameter perturbation, as well as uncertainty in parameter constraint on modeled process activity. Finally, the posterior PDF is presented solely in terms of modeled process activity in order to elucidate the interdependence of modeled microphysical processes under the influence of parameter perturbation and radar observational constraint. 

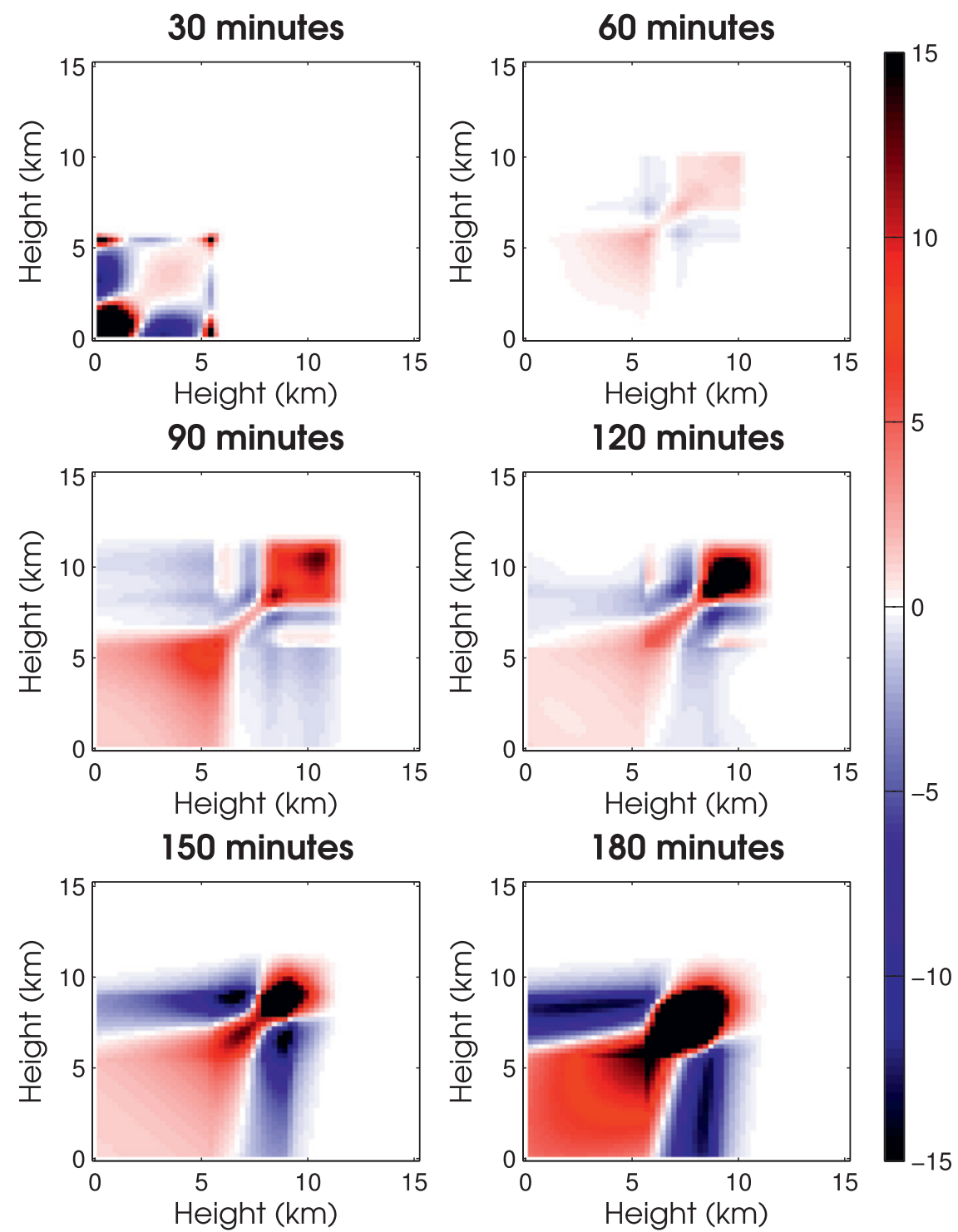

FIG. 3. Radar reflectivity (dBZ) vertical error covariance generated from a $10^{6}$-sample MCMC run using column-integral quantities as observational constraint. Covariance shown for six model time steps. The scale on all plots is -15 to 15 , where blue values denote negative covariance, red is positive, and white is zero.

\section{a. Radar reflectivity error model}

As described in section 2, vertical error covariance in simulated radar reflectivity at each model time is estimated using the model states of the previous MCMC experiment in PV10. Covariance was directly calculated from the $\sim 10^{6}$-member ensemble of radar reflectivity profiles. The results of the covariance calculation not only provide utility as a measure of vertically covarying radar error, but contain detailed information about modeled microphysical behavior, as they are distilled from a very large model ensemble. The error covariance matrix for six model times is shown in Fig. 3. Interpretation of the covariance field is perhaps best understood by considering that covariance between two variables suggests that these variables cannot be considered independent data. Dependence between levels could signify spatial regions where uncertainty is linked via microphysical interaction or constraints supplied by the formulation of the microphysical parameterization scheme. Covariance in the observational error thus acts to reduce the dimensionality of that information space.

While correlation is not immediately indicative of a causal link between variables, it is still instructive to 
consider that covarying features can signal a distinct microphysically relevant behavior within the cloud model. A simple example is visible in Fig. 3 as a prevalent positive covariance for reflectivities in the range between 0 and $6 \mathrm{~km}$ for all time steps after $30 \mathrm{~min}$. Figure 2 of PV10 shows that below $6 \mathrm{~km}$, storm hydrometeors consist virtually exclusively of rain (and cloud water, to which reflectivity at a frequency of $3 \mathrm{GHz}$ is largely insensitive); therefore, a likely explanation of the observed strong lowlevel covariance is a relative lack of activity of microphysical processes within this region. The presence of rain and the associated reflectivity at these levels thus constitutes a single coherent feature, and cannot be viewed as containing independent data. Other notable features of the observational error covariance matrix include strong negative correlation between upper levels (6$10 \mathrm{~km})$ and lower levels $(0-6 \mathrm{~km})$, which is clearly visible as a band of negative values at times 150 and $180 \mathrm{~min}$ and to a lesser extent 60,90 , and $120 \mathrm{~min}$. The extensive and strong negative covariance between upper and lower levels at later model times can be seen as indicating the importance of ice processes (snow, graupel, cloud ice, etc.) in the production of rain in stratiform cloud regimes.

\section{b. Parameter PDFs: Microphysical parameter uncertainty given simulated radar reflectivity observations}

The 10-dimensional posterior parameter PDF is visualized as a series of two-dimensional and one-dimensional marginal PDFs, which are the result of integration across all dimensions except those that form the axes of the marginal. In the case of 2D marginals, integration is performed across 8 dimensions; in the case of 1D marginals, 9. These data are shown in Fig. 4.

The MCMC inversion technique uses comparisons between observations produced by the truth parameters and those produced by the proposal parameters in order to map the PDF of the parameter space. As such, one might expect that the mode (maximum likelihood) of the marginal PDFs would match the truth values for each parameter. While this is predominantly the case for the current study, this result is by no means a foregone conclusion. Indeed, results from PV10 indicate that observations of column integral atmospheric quantities were insufficient to invert the true values of parameters such as snowfall speed exponent, snow, and graupel intercept parameters $\left(b_{s}, N_{0 \mathrm{~s}}\right.$, and $N_{0 \mathrm{~g}}$, respectively). For these parameters the inverse estimate of maximum likelihood in PV10 (the mode of the marginal PDFs) displayed large bias relative to the truth value; for example, the marginal mode of $N_{0 \mathrm{~g}}$ was approximately $800 \%$ larger than truth (see Fig. 6 of PV10). To explain this observation, it is useful to note that because of the process of marginal integration, the location of modes in marginal PDFs need not correspond to their location within the full 10dimensional PDF. In the case of $b_{s}$ in Figs. 6 and 7 of PV10, the mode of the marginal PDF of $b_{s}$ differs greatly from that of the 2D joint marginal PDF of $b_{s}$ and $a_{s}$. Thus, bias observed in the 1D mode of $b_{s}$ is likely a product of marginal integration across the dimension of $a_{s}$. This is not, however, the case for the PDF of $N_{0 \mathrm{~g}}$ in PV10, and alternative explanations must be found. VP08 provide one possible explanation for estimated parameter bias; noting that a Gaussian assumption for observational error can result in bias when the forward model is nonlinear, even if neither the observations nor the model are biased. In both this experiment and PV10, the observational error is assumed to be Gaussian. In the current study, the marginal PDFs of ice hydrometeor intercept parameters, $N_{0 \mathrm{~s}}$ and $N_{0 \mathrm{~g}}$, display the biasing effect of marginal integration as well. The 1D marginal PDFs of these parameters appear to display moderate bias in their maximum likelihood (mode) estimate of the parameter value. Inspection of the joint 2D marginal PDFs of each parameter with snow density, $\rho_{s}$ reveals that the mode of this distribution actually lies much closer to truth than the mode of the 1D marginals, and thus the bias shown in the 1D marginal PDFs can be attributed to the process of marginal integration across the dimension of $\rho_{s}$. Regardless, the current results with radar reflectivity suggest much less bias in the location of the maximum likelihood with respect to reference truth than the column integral observations of PV10, thus confirming a more unique inversion using these observations.

The first-order measure of parameter uncertaintythe standard deviation (or analogously, the variance) of the PDF-is shown to be greatly reduced when using radar reflectivity as the observational constraint (see Table 2). Parameters that strongly control simulated radar reflectivity are well constrained by the observations and exhibit relatively less variance than parameters that have little effect on the radar profile. For example, within the range of allowed values, all parameters besides $q_{\mathrm{c} 0}$, the cloud mixing ratio autoconversion threshold, are better constrained by radar reflectivity than columnintegral observations. The $q_{\mathrm{c} 0}$ is comparatively poorly constrained because of the presence of a secondary mode at values near the upper range of this parameter. In particular, the parameters related to the particle size distribution (the intercept parameters $N_{0 \mathrm{x}}$ ) are highly constrained by radar reflectivity observations, with $N_{0 \mathrm{~g}}$ experiencing a reduction in standard deviation of nearly an order of magnitude. This reduction in parameter uncertainty highlights the increased information content of radar reflectivity relative to column-integral measurements, vis-à-vis cloud microphysical parameters. While 


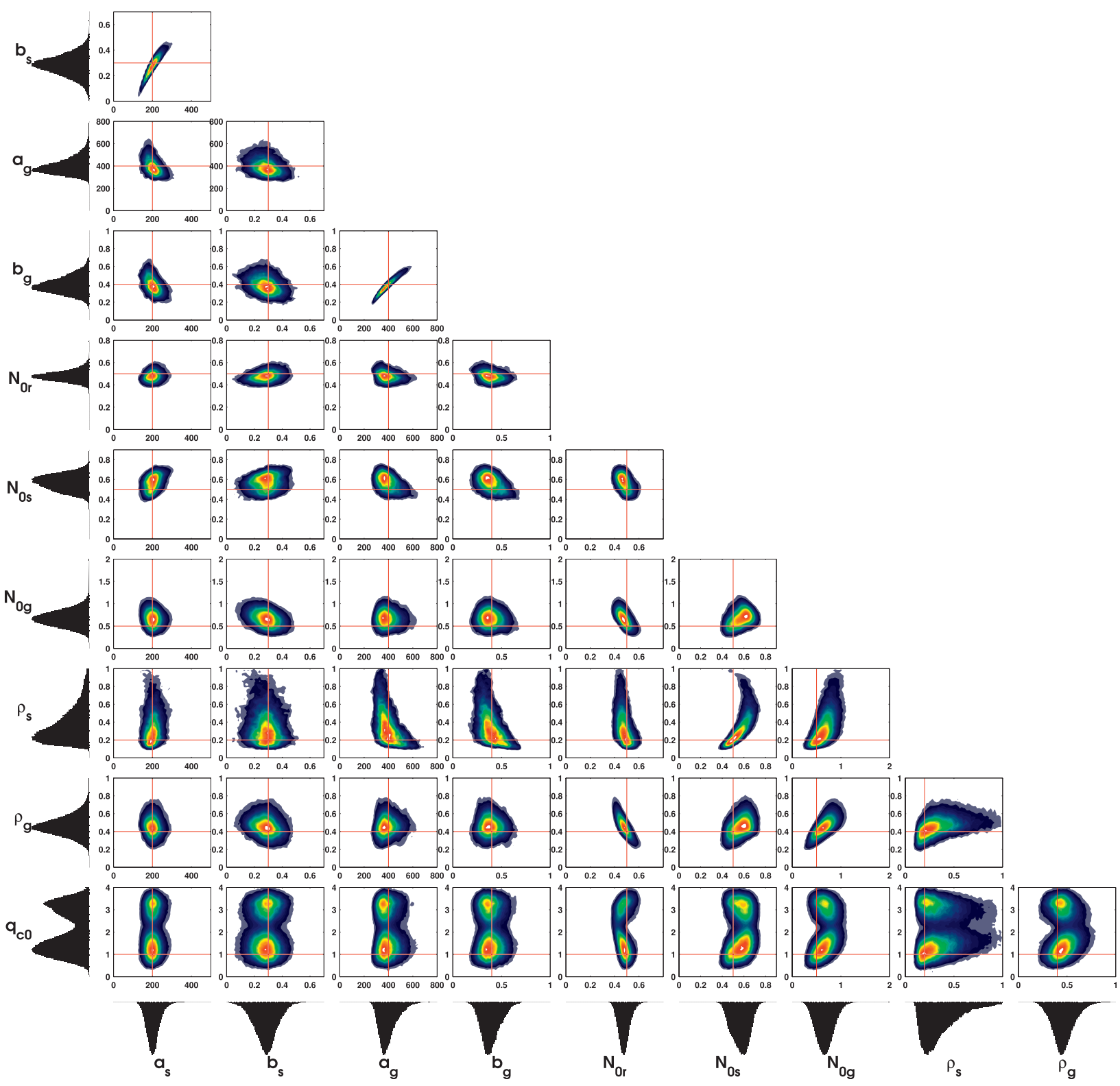

FIG. 4. Posterior parameter PDF displayed as joint 2D marginal distributions for each pair of parameters and 1D marginals for each individual parameter. Observational constraint is profiles of simulated radar reflectivity taken at 60 and 120 min into the model run. In each plot, dark colors represent regions of relatively low probability density, whereas lighter and warmer colors represent regions of relatively high probability density. Outer white regions denote $\approx 0$ probability. The solid red lines mark the locations of the parameter truth values.

this is an expected result, it should not be treated as a foregone conclusion, given the fact that reflectivity is nonuniquely related to the intercept parameter of particle size distribution of hydrometeors. Thus, it cannot be assumed a priori that there will not be multiple modes in the PDF of $N_{0 \mathrm{x}}$ parameters.

The 2D marginals may also be inspected for evidence of parameter inter-independence, nonlinearity, and multimodality. Interdependence of parameters would suggest a reduction in the dimensionality of the control parameter space (i.e., parameters whose values are dependent on the value of another parameter). Nonlinearity, whether unimodal or multimodal, can be viewed as skewness or asymmetry in the marginals and may present difficulties for the solution of data assimilation and inverse modeling problems, especially when linear or Gaussian assumptions are made (VP08). Multimodality in the PDF indicates the fact that multiple combinations of parameter 
TABLE 2. Standard deviation of each posterior marginal parameter PDF for the inverse experiment utilizing column-integral observations (PV10), and the inverse experiment utilizing vertically resolved radar reflectivity observations (this study).

\begin{tabular}{ccc}
\hline \hline Parameter & Std dev (PV10) & Std dev (this study) \\
\hline$a_{s}$ & 245.2 & 31.7 \\
$b_{s}$ & 0.2462 & 0.0809 \\
$a_{g}$ & 241.2 & 63.3 \\
$b_{g}$ & 0.1453 & 0.0814 \\
$N_{0 \mathrm{r}}$ & 0.1786 & 0.0468 \\
$N_{0 \mathrm{~s}}$ & 1.377 & 0.0739 \\
$N_{0 \mathrm{~g}}$ & 1.355 & 0.1782 \\
$\rho_{s}$ & 0.240 & 0.1940 \\
$\rho_{g}$ & 0.2713 & 0.1146 \\
$q_{\mathrm{c} 0}$ & 0.6933 & 0.950 \\
\hline
\end{tabular}

values produce realistic model output. When diagnosing these features of the PDF, it is crucial to note that the shape of the 10-dimensional PDF may not be reflected by that of its marginals, and the process of integration across the other parameters may have the effect of obscuring multiple modes or locally nonlinear regions of the PDF. This is evident in Figs. 8 and 9 of PV10, where the joint 2D marginal of $a_{g}$ and $b_{g}$ displays two modes, whereas the 1D marginals of these parameters (Fig. 8 of PV10) show unimodal distributions. That particular multimodal feature is not evident in the current inversion with radar reflectivity, further indicating stronger constraint on the parameter values by these observations.

Interdependence between parameters can be observed via the combined space of these parameters as represented in 2D marginals PDFs shown in Fig. 4. Strong covariance can be seen in the 2D marginal PDF of $a_{s} / b_{s}$, the mass-weighted snowfall speed coefficient and exponent parameters. This indicates that uncertainty in these parameters is essentially bivariate, with variance in the PDF caused entirely by covariance of these two variables. Considering that these parameters affect fall speed via the relationship $V_{s}\left(D_{s}\right)=a_{s} D^{b_{s}}$, it is likely that these parameters covary so as to minimize changes in fall speed.

Multimodality is evident in Fig. 4-the marginal PDF for $q_{\mathrm{c} 0}$ displays a primary mode near the truth value of $1.0 \mathrm{~g} \mathrm{~kg}^{-1}$ as well as a secondary mode near a value of $3.4 \mathrm{~g} \mathrm{~kg}^{-1}$. This is curious considering the role that $q_{\mathrm{c} 0}$ plays in the microphysics parameterization scheme; $q_{\mathrm{c} 0}$ is the mixing ratio threshold at which cloud water will autoconvert to rain. As will be shown in section 3d, the mode with the greater parameter value indicates an observationally consistent model solution involves strongly suppressed (perhaps negligible) autoconversion of cloud water to rain. Inspection of the $2 \mathrm{D}$ marginals shows that the secondary mode is not correlated with a change in any of the other 9 parameters; that is, in each of the 2D marginals with $q_{\mathrm{c} 0}$, the location of both modes is the same for the other parameter considered. What this suggests is that both modes of $q_{\mathrm{c} 0}$ can produce realistic observations without any "special" perturbation of the other parameters. That said, within each mode of $q_{\mathrm{c} 0}$, covariance with other parameters changes sign-an effect particularly evident in the $2 \mathrm{D}$ marginals with intercept parameters and ice particle densities $\left(N_{0 \mathrm{r}}, N_{0 \mathrm{~s}}, N_{0 \mathrm{~g}}, \rho_{s}\right.$, and $\left.\rho_{g}\right)$. This is discussed further in the following sections where joint PDFs of reflectivity and parameters and microphysical processes and parameters are presented. It should be noted that the accumulated marginal value (a proxy for likelihood) for the primary mode of $q_{\mathrm{c} 0}$ is greater than for the secondary mode, indicating that even though extreme values of $q_{\mathrm{c} 0}$ are capable of producing somewhat realistic observations (via compensating mechanisms discussed in the next section), those observations are nonetheless farther from truth than for values of $q_{\mathrm{c} 0}$ in the region of the primary mode.

The following sections extend the probabilistic analysis of parameter PDFs to distributions of simulated observations as well as individual microphysical process activities. The joint parameter-observation PDFs (section $3 \mathrm{c}$ ) reveal the combined information space of control parameters and observations, illuminating how the former is constrained by the latter as well as the sensitivity of the latter to the former. The activity of individual microphysical processes is presented in section $3 \mathrm{~d}$ as a joint PDF with the control parameter values. This data indicates how perturbations in the control parameters affect the activity of modeled microphysical behavior, illuminating the processes that act to change the structure of the observed storm. Finally, in section $3 e$ the results are presented fully in the space of microphysical processes to reveal the relationships between individual modeled microphysical processes.

\section{c. Parameter-observation PDFs: Sensitivity of a vertically resolved observation to microphysical parameter perturbation}

The Markov chain generated in a MCMC experiment allows for the probabilistic treatment of any modelgenerated variable. For every instance of the Markov chain, the simulated observation value forms a pair with the parameter value, generating a joint observationparameter PDF. This is analogous to Figs. 10 and 11 of PV10, where the multiple, column integral observation types are used. In the current analysis the two instances of radar reflectivity observation are used: one during the convective phase of the squall-line simulation and one during the stratiform phase (at 60 and $120 \mathrm{~min}$ into the simulation time, respectively). The PDFs for convective 


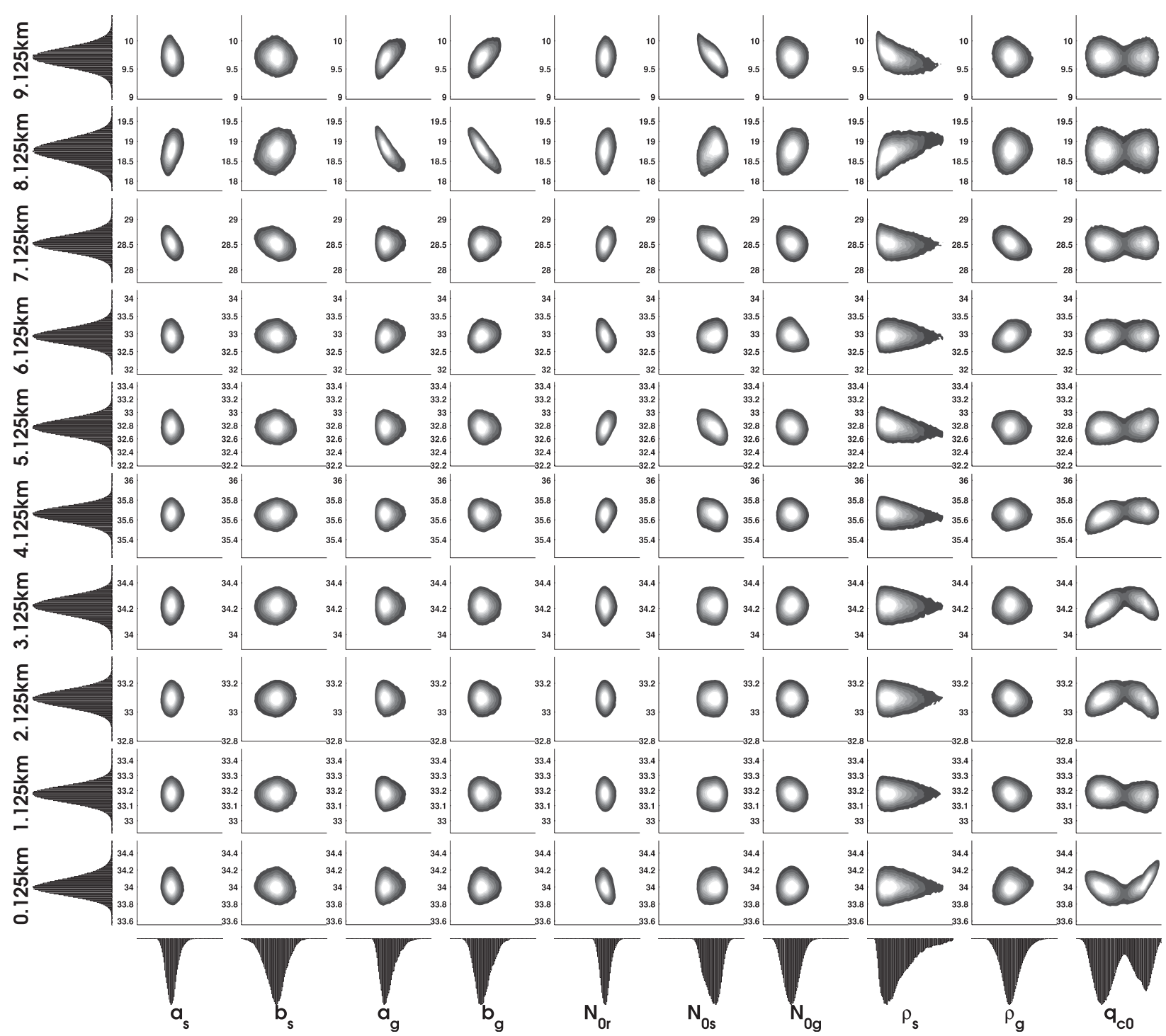

FIG. 5. Joint posterior PDFs of parameters and simulated radar reflectivity observations at various vertical levels for a time during the convective phase of squall-line development (60 min into the simulation). Each row corresponds to simulated reflectivity at a particular model level, whereas each column corresponds to a different model physics parameter.

observations (Fig. 5) and stratiform observations (Fig. 6) are presented separately, allowing an examination of how parameter-observation relationships differ in distinct modeled physical environments. As with the parameter PDFs, 2D and 1D marginals are presented. In addition, the first-order relationship between parameter and radar reflectivity is presented in terms of covariance between these values (Figs. 7 and 8 for the convective and stratiform regimes, respectively). The simulated radar reflectivity observations are vertically resolved at model resolution and thus each profile consists of 62 data that are effectively separate observations. For convenience 10 observation levels out of the 62 are chosen as representative of the variability observed in the full profile. These data correspond to observations of the model at every kilometer between 0.125 and $9.125 \mathrm{~km}$.

\section{1) JOINT PARAMETER-OBSERVATION PDFs: CONVECTIVE}

The joint 2D marginals of microphysical parameters and reflectivity observation values represent the full relationship between these variables. This includes the first-order relationship in addition to any higher-order nonlinearities and multimodal properties. Only parameter-observation pairs that show linear or Gaussian characters will be well diagnosed by the covariance shown in Figs. 7 and 8. For the convective regime, the 2D marginal PDFs in Fig. 5 are all roughly 


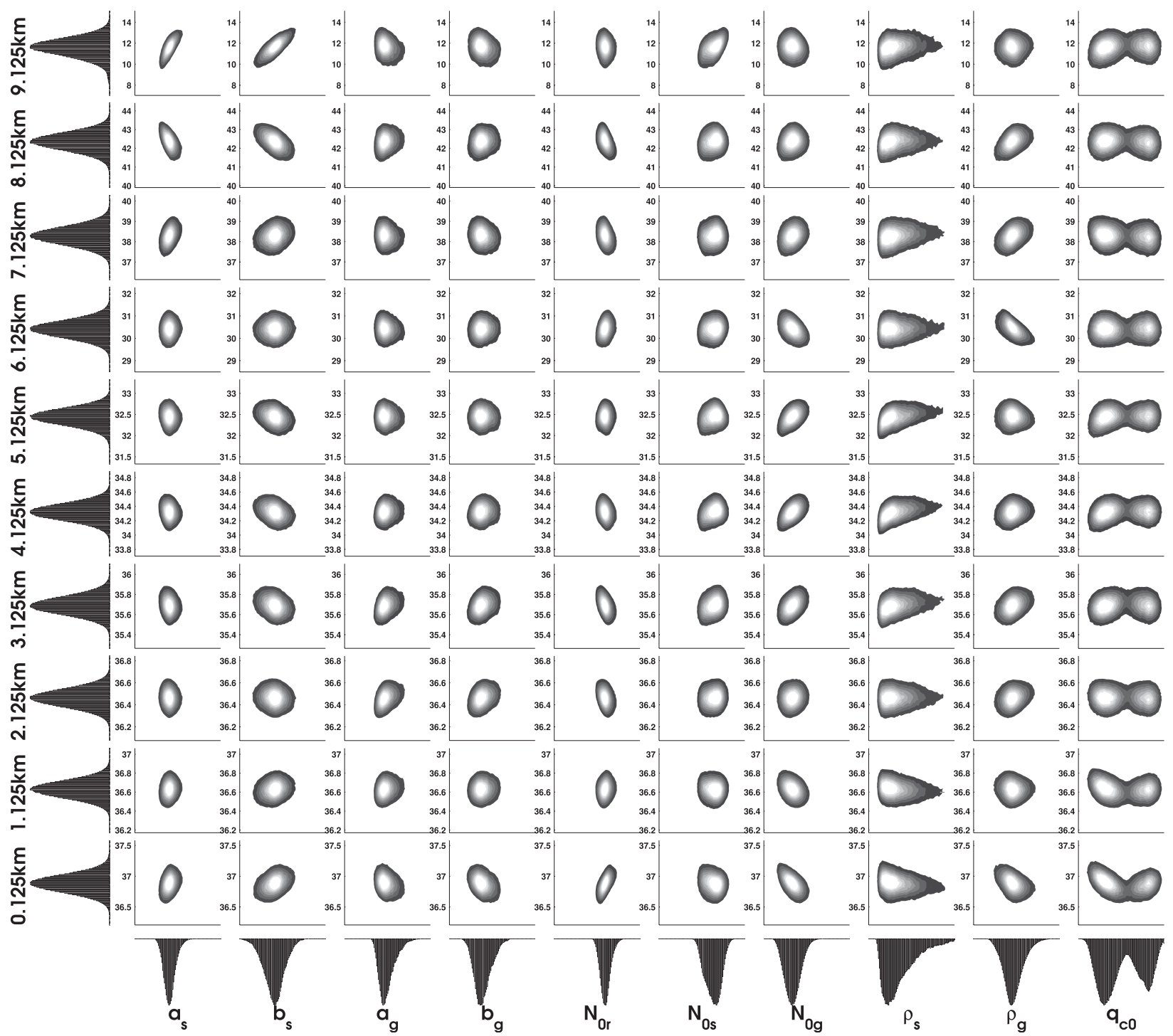

FIG. 6. As in Fig. 5, but for a time during the stratiform phase of squall-line development (120 min into the simulation).

Gaussian with the notable exception of $\rho_{s}$ and $q_{\mathrm{c} 0}$, which display nonlinearity and multimodality, respectively.

Many of the features shown in Fig. 5 have simple physical explanations. As might be expected, observations at model levels above the freezing line (approximately at a height of $6 \mathrm{~km}$ ) are sensitive to perturbations in graupel fall speed parameters $a_{g}, b_{g}$, and to a lesser extent snowfall speed parameters $a_{s}$ and $b_{s}$. These parameters control the fall speed of graupel and snow, and thus can be expected to be influential in determining the vertical distribution of reflectivity when snow and graupel are formed in a strong convective updraft. This effect is clearly visible in the 2D marginals of $a_{g}$ and $b_{g}$ with observations at 8.125 and $9.125 \mathrm{~km}$ in Fig. 5 as well as the 2D marginals of $a_{s}$ and $b_{s}$ with observations between 7.125 and $9.125 \mathrm{~km}$. By arguments similar to those above, the shapes of 2D marginals for rain parameters $N_{0 r}$ and $q_{\mathrm{c} 0}$ can be rationalized. Rain parameters have the greatest effect on observations in nonfreezing regions (i.e., below $6 \mathrm{~km}$ ). It can be observed that $N_{0 \mathrm{r}}$ has the most noticeable impact on observations just below $6 \mathrm{~km}$, whereas $q_{\mathrm{c} 0}$ appears to take over at the lowest model levels between 0 and $4 \mathrm{~km}$. It is possible that the observed differences between rain parameters is related to the perturbation of accretion processes, which may be more closely related to $N_{0 \mathrm{r}}$ than $q_{\mathrm{c} 0}-\mathrm{a}$ discussion of this conjecture will be given in section 3d. Comparison of Fig. 5 with parameter PDFs in Fig. 4 also yields insight. Parameter pairs $a_{s} / b_{s}$ and $a_{g} / b_{g}$ are shown in Fig. 4 to be strongly dependent and so the shapes of 2D marginals (and thus, the signs of the covariances) in Fig. 5 are generally similar for these parameter pairs. 


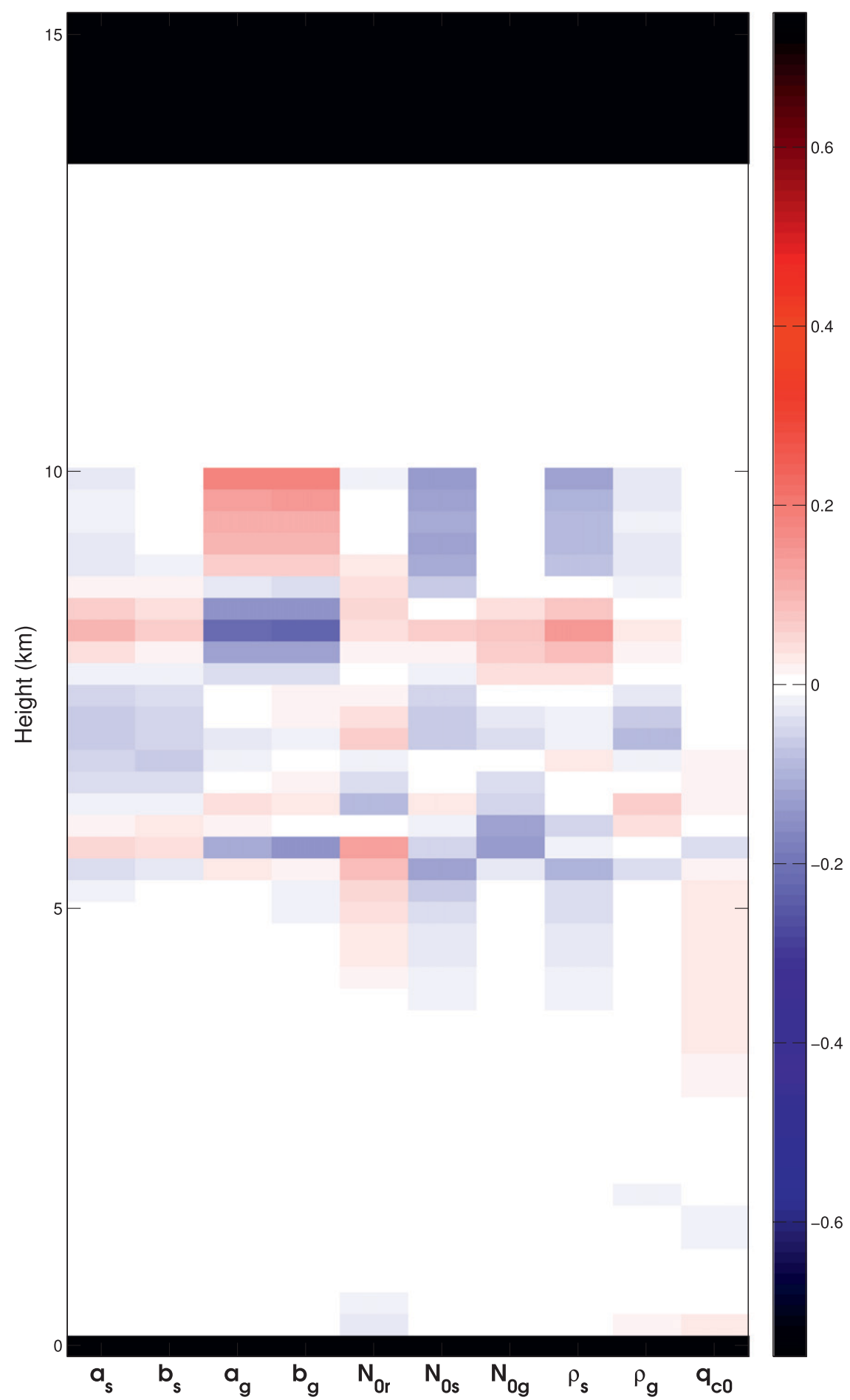

FIG. 7. Covariance for joint observation-parameter PDF 2D marginals. Parameters are on the abscissa and vertical level of simulated radar observation are on the ordinate. Covariance is normalized by parameter standard deviation. Observations are for convective regime of squallline simulation $(60 \mathrm{~min})$. 


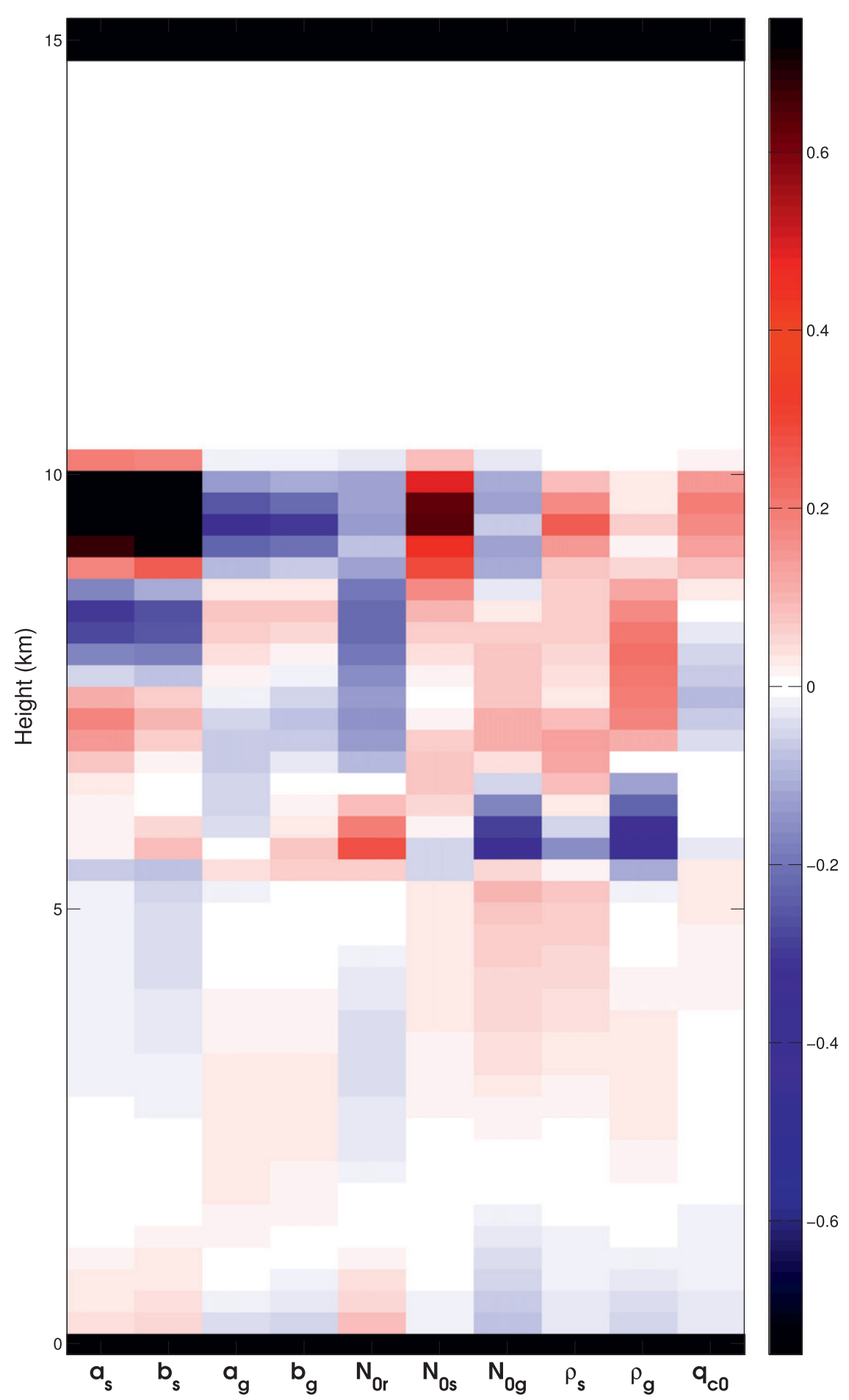

FIG. 8. As in Fig. 7, but during the stratiform phase of squall-line development (120 min into the simulation).

An interesting feature observed in many of the 2D parameter-observation marginals is that first-order relationships between observations and parameters changes sign with height. This effect is evident in Fig. 5, where for the parameters $a_{g}, b_{g}, N_{0 \mathrm{~s}}$, and $\rho_{s}$, changes in the tilt of the PDF are observed between levels 8.125 and $9.125 \mathrm{~km}$. The observed change in sign of parameterobservation first-order relationships can be interpreted as 
a sign that higher reflectivity at one level comes at the expense of lower reflectivity at another level-a natural result of perturbing parameters such as $a_{g}$ and $b_{g}$, which affect the fall speed and sedimentation and thus vertical distribution of hydrometeors. The first-order relationship of covariance is further discussed in section $3 c(3)$.

In addition to correlations between parameter and observation value, the joint marginals in Fig. 5 provide insights into parameter-observational uncertainty, which is non-Gaussian-in particular the PDFs of $\rho_{s}$ and $q_{\mathrm{c} 0}$. The 1D marginal PDF of $\rho_{s}$ has considerable skewness, with a sharp decrease in probability at low values of the parameter. It is apparent from the 2D joint marginal PDFs with observations at 8.125 and $9.125 \mathrm{~km}$ that this is associated with sharply varying reflectivity values at these lower parameter values, compared with relatively constant reflectivity values at higher values of $\rho_{s}$. Note also the relative insensitivity of observation to variations in $\rho_{s}$ for vertical levels other than those just mentioned-again emphasizing the strong height dependence of radar observational information. It is likely that the observed relationships for $\rho_{s}$ are a product of the inverse relationship between density and particle surface area, which is directly related to fall speed and accretion cross section (Posselt 2006). This hypothesis is discussed further in section $3 \mathrm{~d}$.

The other strongly nonlinear parameter marginal belongs to the cloud water-to-rain autoconversion threshold parameter $\left(q_{\mathrm{c} 0}\right)$, which displays a bimodal structure. In section $3 b$ it was noted that the separation in the two modes of $q_{\mathrm{c} 0}$ is not correlated with other parameter values. Figure 5 shows that the separation in modes of $q_{\mathrm{c} 0}$ is, however, correlated with modified observational values and furthermore, with changes in the relationship between parameter perturbation and observed reflectivity. For example, the secondary (higher $q_{\mathrm{c} 0}$ ) mode appears to display greater reflectivity than the primary mode for levels 3.125 to $5.125 \mathrm{~km}$. This difference in reflectivity between primary and secondary modes may help to explain why the secondary mode of $q_{\mathrm{c} 0}$ displays reduced likelihood. These PDFs also illustrate that the bimodality displayed is directly related to a nonmonotonic relationship between simulated radar reflectivity and the parameter $q_{\mathrm{c} 0}$. This effect is most clearly seen for radar observations at $0.125,2.125$, and $3.125 \mathrm{~km}$, where values of $q_{\mathrm{c} 0}$ between the modes produce excessively high or low values of simulated radar reflectivity.

\section{2) JOINT PARAMETER-OBSERVATION PDFS: STRATIFORM}

Comparison of Fig. 6 with Fig. 5 shows many previous conclusions about ice hydrometeor parameters are still relevant: the greatest covariance with observation occurs at higher levels-particularly between 7.125 and $9.125 \mathrm{~km}$ for $a_{s} / b_{s}$ and $9.125 \mathrm{~km}$ for $N_{0 \mathrm{~s}}$. Additionally, however, significant covariance is observed for ice parameters at lower levels, indicating the strengthened role of ice parameters on controlling rain rate within the stratiform regime. For example, $a_{g} / b_{g}$ displays moderate positive correlation with reflectivity between 0.125 and $4.125 \mathrm{~km}$, demonstrating that increased values of these parameters (related to graupel fall speed) are associated with increased lower-level reflectivity. A weakly negative correlation is observed, however, between $a_{s} / b_{s}$ and reflectivity between 3.125 and $5.125 \mathrm{~km}$. These effects highlight the complicated relationship between ice hydrometeors within the stratiform rain region of the simulated storm. It is possible that accretion processes, which are strongly controlled by fall speed, are responsible for the observational sensitivity at these levels. Another possibility is that other processes sensitive to snowfall speed, such as snow melting processes, contribute; or that the effect shown here is a result of a complex interaction between multiple microphysical processes and hydrometeor types. One attempt at a diagnosis of these effects is presented in section $3 \mathrm{~d}$.

In the previous section, the nonlinear relationship between radar reflectivity and snow density $\rho_{s}$ was cited as a possible cause of the skewness observed in the marginal of $\rho_{s}$. For the stratiform regime, we observe in the 2D marginals of $\rho_{s}$ similar features, with the most notable change being that these features are observed throughout the atmospheric column, whereas during the convective phase they are largely limited to regions above $7.125 \mathrm{~km}$. The other parameter displaying strong nonlinearity in its marginal PDF, $q_{\mathrm{c} 0}$, maintains many of the features observed in the convective regime. In general, the nature of the relationship between parameter perturbation and reflectivity in this case is unchanged. One notable difference, however, is that the two modes of $q_{\mathrm{c} 0}$ do not appear to be associated with modified values of radar reflectivity. This suggests that the constraint that results in reduced likelihood for the secondary mode of $q_{\mathrm{c} 0}$ occurs largely within the convective regime of the storm. This result is consistent with the greater role that autoconversion of cloud water has within the convective regime.

\section{3) PARAmeter-ObServation COVARIANCE}

Covariance of parameter and observation values are presented in order to display the first-order relationships with greater clarity and to facilitate relative comparison between observational data and parameter perturbations. In this case, the interest is not in the variance in parameter value (which is arbitrary for the purpose of comparison between parameters), so the covariance is 
normalized by parameter standard deviation. Normalization by reflectivity standard deviation is not performed, however, in order to preserve a measure of the degree to which observations are affected by parameter uncertainty. Note also that covariance carries the most information for PDFs, which are closest to Gaussian in structure. Strongly nonlinear or multimodal PDFs, such as those of $\rho_{s}$ and $q_{\mathrm{c} 0}$ will in particular be poorly characterized by covariance. Normalized covariance is displayed in Figs. 7 and 8, with parameters on the abscissa and height of simulated reflectivity observation on the ordinate. Strong values indicate observations that are highly sensitive to perturbations in a particular parameter. Only if an observation is sensitive to parameter perturbations can it serve as a constraint in the inversion of that parameter. Thus, these figures serve to plot the observational regions that can possibly serve as first-order model constraint within the inverse experiment.

For the convective regime, shown in Fig. 7, strong covariances are observed at the freezing level $(\approx 6 \mathrm{~km})$, suggesting that even in the absence of a simulated radar melting layer bright band, melting-freezing effects are integral to microphysical processes. The strongest relationships here are observed for graupel fall speed parameters $a_{g}$ and $b_{g}$; PSD intercept parameters $N_{0 \mathrm{r}}, N_{0 \mathrm{~s}}$, and $N_{0 \mathrm{~g}}$; and snow density $\rho_{s}$. The highest values of covariance for $a_{g}$ and $b_{g}$ are observed between 8 and $10 \mathrm{~km}$. This is likely associated with strong updrafts at this observational time (PV10 see their Fig. 1c), which act to suspend ice particles at high levels. It is only at these high levels, where vertical velocity forcing decreases, that ice fall speed parameters can strongly affect ice hydrometeor concentrations, and thus reflectivity. This suggests that during the convective regime storm behavior is more strongly determined by dynamical forcing (here in the form of prescribed, time-varying profiles of updraft and water vapor) than by microphysical processes. Snow PSD intercept parameter $N_{0 \mathrm{~s}}$ and density $\rho_{s}$ display a similar, albeit opposite, pattern in values of covariance at upper levels, demonstrating the strong constraint by snow processes on reflectivity within this storm region. Strong positive covariance is observed for $N_{0 \mathrm{r}}$ at levels just below the freezing level. Note that increased values of $N_{0 \mathrm{r}}$ indicate a particle size distribution that favors small droplets over large. This would suggest enhanced evaporation and lower fall speed, possibly favoring accretion processes, which require fall speed differential between species. In section $3 \mathrm{~d}$ these relationships are explicitly investigated.

From Fig. 8 it is clear that observational covariance with parameter perturbation is generally greater for the stratiform regime than the convective regime. This result is expected, and can be observed in the increase in observational uncertainty as represented by observational error covariance shown in Fig. 3. This result is analogous to the increase in model error with time observed in the simulation of most complex, nonlinear systems. Another explanation for this effect is, as mentioned in the previous paragraph, the constraint on storm behavior provided by the prescribed forcing - it is possible that during the stratiform regime, microphysical process constraint is more dominant than during the convective regime. In addition to this overall increase in covariance, differences are observed in the vertical structure of parameter-observation covariance between the convective and stratiform regimes. As observed in Fig. 6, ice parameters have as great of an impact on lower-level observational variance as rain parameters, indicating the importance of ice microphysics on stratiform rain. More specific conclusions can be drawn as well. For example, snowfall speed parameters $a_{s}$ and $b_{s}$ display very strong covariances at upper storm levels (between 8 and $10 \mathrm{~km}$ ), indicating the importance of fall speed calculation on the vertical distribution of stratiform reflectivity. Note that while increased values of these parameters strongly increase reflectivity between 9 and $10 \mathrm{~km}$, it strongly reduces reflectivity between 8 and $9 \mathrm{~km}$ and slightly reduces it in the rain region below $6 \mathrm{~km}$. This points to the importance of sedimentation as well as processes that relate rain and snow in the stratiform regime of the modeled cloud such as melting or accretion processes. The effect of parameter perturbations on simulated observations can be further illuminated by considering the effect of parameter perturbation on individual modeled microphysical processes, which is discussed in the following section.

\section{d. Parameter-microphysical process PDF: Microphysical process constraint and sensitivity to parameter perturbations}

The equations that govern modeled microphysical processes, produce hydrometeors, and describe their interaction are, with negligible exception, deterministically related to parameters and model state. The deterministic relationships between parameters and processes are inadequate for the purposes of ascertaining how microphysical process activity is related to microphysical parameter uncertainty within a simulated storm. Simple inspection of these equations reveals that processes are nonlinearly related to microphysical parameter values and model state. Even a process as simple as sedimentation of snow depends on four parameter values- $a_{s}, b_{s}, \rho_{s}, N_{0 \mathrm{~s}}$-as well as snow mixing ratio. Accretion processes are dependent not only on 
microphysical parameters, but the mixing ratios of multiple hydrometeor species and their fall speeds, which are themselves a nonlinear product of antecedent model state and parameter values. MCMC allows for a probabilistic determination of the realistic range of the relationship between microphysical parameters, uncertainty in these parameters, and the processes they help to control.

Just as in the case of parameter-observation joint PDFs, the Markov chain provides an ensemble that represents the likelihood of a model state with respect to some observation. From each model run in the Markov chain, a vertically and temporally integrated measure of microphysical process activity is extracted to obtain a PDF of microphysical processes. Temporal integration is performed across the convective phase of the squallline simulation (50-70 $\mathrm{min}$ ) as well as the stratiform phase (110-130 min); as with parameter-observation PDFs, these joint PDFs are presented separately. The joint parameter-process PDFs are represented as 1D and 2D marginals in Fig. 9 (convective) and Fig. 10 (stratiform). The bulk microphysical parameterization scheme used here simulates the contribution from over 40 processes-a number too great to examine within the scope of this paper. The number is culled down to the 16 processes with the greatest variance in their contribution across the MCMC ensemble. These 16 processes and the abbreviations used to label them are listed in Table 3. Full interpretation of the 2D marginals even with a reduced set of processes is beyond the scope of this study. The discussion that follows is limited to a few of the most prominent results.

If the activity of a given microphysical process depends solely on the value of a microphysical parameter (this is highly unlikely), then the 2D marginal is expected to collapse to a line displaying the deterministic relationship between these two variables. If there is uncertainty in the process's dependence on that parameter, or if the process depends on more than one parameter, this deterministic line will be broadened. In the case of current study, parameters have an uncertainty that is the result of their interaction with other perturbed parameters within a nonlinear model; processes too have uncertainty related to parameter uncertainty in addition to uncertainty in the model state, which is itself a function of the parameters. As such, for most processes considerable deviation from a deterministic line is expected. A microphysical process may also be insensitive to variations in some parameter. In such a case the 2D marginal will approach the appearance of a 2D Gaussian with covariance $=0$. More complex shapes may indicate nonlinearities in microphysical process response to parameter uncertainty.

\section{1) PARAMETER-MICROPHYSICAL PROCESS PDFS: CONVECTIVE}

In Fig. 9 several of the 2D marginals display variance in modeled process activity that is dominated by covariance with a parameter. In many of these cases, the relationship between parameter and microphysical process is unsurprising - both parameter and process are related to the same hydrometeor type and thus there is relatively little uncertainty in the relationship between process and parameter. For example, the activity of processes related to rain such as ERN, PIACR, PRACW, and QRACS is strongly dependent on the value of the rain size distribution intercept parameter, $N_{0 r}$; processes related to snow such as PSACR, PSDEP, PSACW, PSFW, and PSFI show similarly strong constraint by snow density $\rho_{s}$ and, to a lesser extent, $N_{0 s}$. Graupel processes such as PGMLT, DGACR, QGACW, and PGDEP show some dependence on graupel parameters $N_{0 \mathrm{~g}}$ and $\rho_{g}$, but not to the degree that is shown by snow and rain processes. Fall speed parameters $a_{s}, b_{s}, a_{g}$, and $b_{g}$ seem to control processes in a more indirect manner than both particle size distribution and density parameters $\left(N_{0 \mathrm{x}}\right.$ and $\left.\rho_{x}\right)$-there is greater uncertainty in the relationship between process and parameter here.

The parameter-process PDFs have the ability to help illuminate one of the most vexing features shown in the parameter PDFs of Fig. 4: the bimodal structure of cloud water autoconversion threshold, $q_{\mathrm{c} 0}$. The relevant question is whether the separation in the modes of $q_{\mathrm{c} 0}$ is reflected in distinct values of process activity and in particular, if some processes compensate for perturbed activity in other processes. The most apparent differences in process activity for each mode are shown by the evaporation of rain, ERN, autoconversion of cloud water to rain, PRAUT, graupel accretion of cloud water, DGACW, and ice accretion of rain, PIACR. The 2D marginals of $q_{\mathrm{c} 0}$ in Fig. 9 show that the upper mode of $q_{\mathrm{c} 0}$ is associated with increased evaporation of rain, decreased autoconversion of cloud water to rain, increased graupel accretion of cloud water, and decreased ice accretion of rain. Thus, the increased activity of DGACW serves to partially offset any increased cloud water concentrations associated with a strongly suppressed cloud water autoconversion. Likewise, decreased activity of PIACR acts to offset any decreased rain concentrations produced by enhanced evaporation and decreased autoconversion. These compensating processes serve to produce a model state that is not observationally distinct from the truth.

The 2D joint marginal PDFs of rain evaporation, ERN, with parameters displays a change in its relationship with parameter values between the upper and lower modes of 
$188886888 \%$

$=0000000000$

$\angle 0000100 \Delta \Delta \Delta$

$\angle 0000000000$

$<000000000 \infty$

$\angle 0000000000$

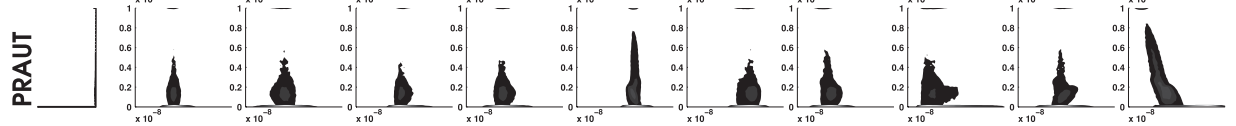

240000100000

5000000000

$\angle 0000100000$

2400001000

10000010000

$<000000 \% 00$

$40000000 \% 00$

20000000000

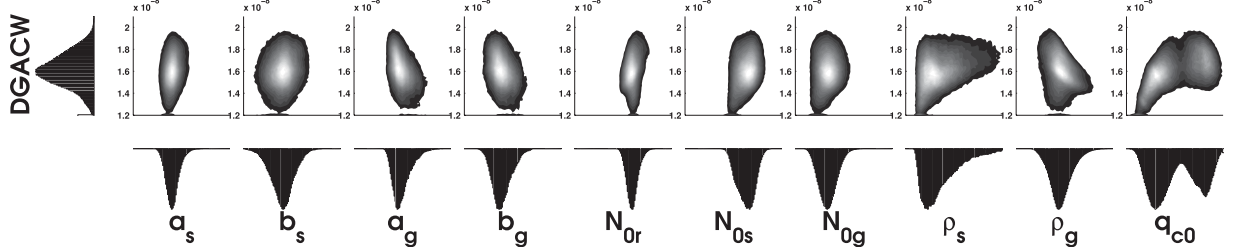

FIG. 9. Joint posterior PDFs of parameters and microphysical process activity during the convective phase of squall-line development (50-70 min into the simulation). Each row represents a spatiotemporal integral of process activity (listed in Table 3 ), each column represents a microphysical parameter. 

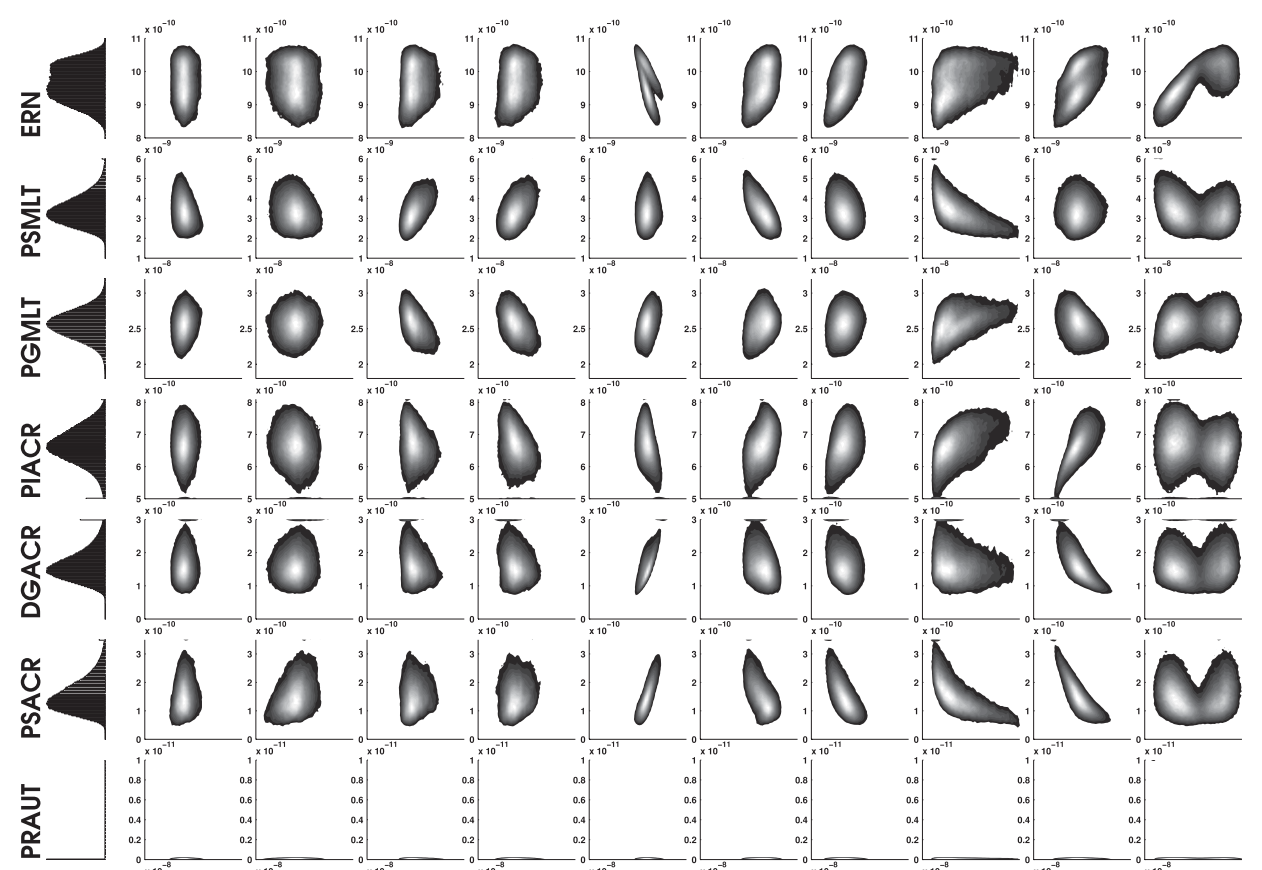

- 000100000 . 总 00000000 : $\quad 000000000$ \begin{tabular}{llllllllll}
\hline & 0 & 0 & 0 & 0 & 0
\end{tabular} 竞 0000000000

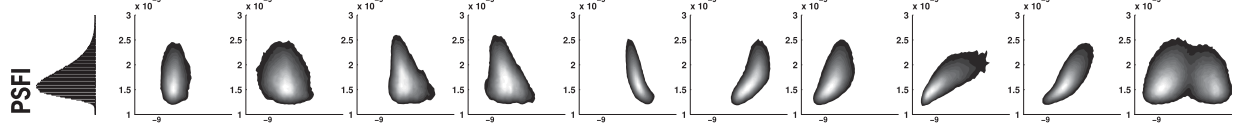

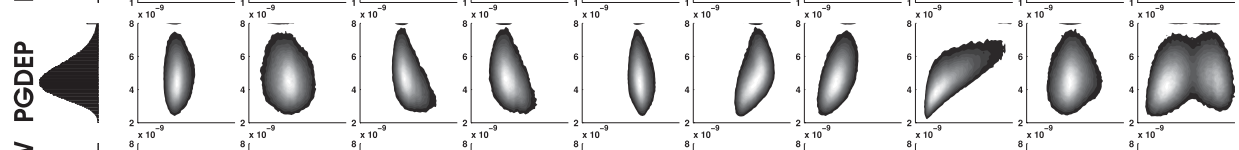

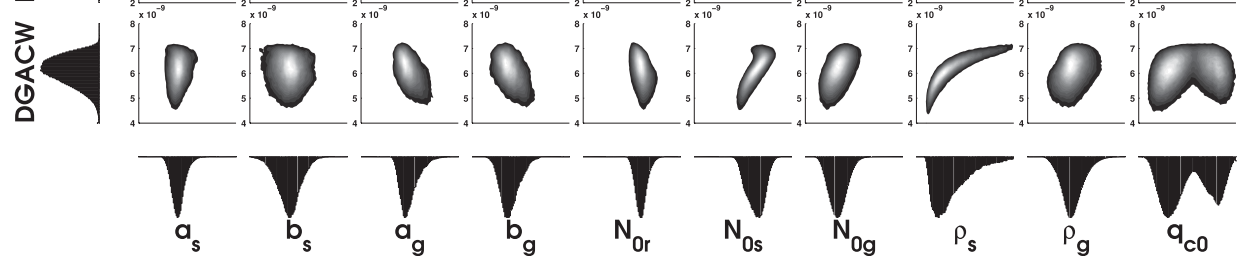
FIG. 10. As in Fig. 9 but during the stratiform phase of squall-line development (110-130 min into the simulation). 
TABLE 3. Microphysical process descriptions, abbreviation, source, and product hydrometeors of the process. Note that ERN, the evaporation of rain, is a negative definite quantity and here only the absolute value is shown.

\begin{tabular}{llll}
\hline \hline \multicolumn{1}{c}{ Process description } & Abbreviation & \multicolumn{1}{c}{ Source } & Product \\
\hline Evaporation of rain (negative) & ERN & Rain & Water vapor \\
Melting of snow & PSMLT & Snow & Rain \\
Melting of graupel & PGMLT & Graupel & Snow, graupel \\
Cloud ice accretion of rain & PIACR & Cloud ice, rain & Graupel \\
Graupel accretion of rain & DGACR & Graupel, rain & Snow, graupel \\
Snow accretion of rain & PSACR & Rain, snow & Rain \\
Autoconversion of cloud water to rain & PRAUT & Cloud water & Rain \\
Rain accretion of cloud water & PRACW & Rain, cloud water & Rain \\
Graupel accretion of cloud water to produce rain & QGACW & Graupel, cloud water \\
Rain accretion of snow & QRACS & Rain, snow & Rain \\
Deposition on snow & PGDEP & Water vapor, snow & Snow \\
Snow accretion of cloud water & PSACW & Cloud water, snow & Snow, graupel \\
Bergeron process (deposition/riming) & PSFW & Cloud water & Snow \\
Deposition on graupel & PGDEP & Water vapor & Graupel \\
Graupel accretion of cloud water & DGACW & Graupel, cloud water & Graupel \\
\hline
\end{tabular}

this process's PDF. In the case of ERN and $N_{0 \mathrm{r}}$, for example, the upper modes of the 2D marginal displays a strongly positive covariance between parameter and process activity, whereas the lower mode shows little obvious covariance-indicative of uncertainty in the relationship between the parameter $N_{0 r}$ and ERN. Similar behavior is shown between ERN and graupel density $\rho_{g}$, and to a lesser extent, snow and graupel intercept parameters $N_{0 s}$ and $N_{0 \mathrm{~g}}$. This suggests that microphysical process activity is strongly dependent on the cloud microphysical state; that is, that the relationship between process and parameter and the uncertainty in that relationship can vary greatly depending on variations in other parameter values (or as will be seen in the next section, depending on variations in storm morphology).

Observed nonlinearities in the parameter-parameter PDFs can also be diagnosed. For example, the marginal for snow density $\rho_{s}$ has notable skewness, with a sharp drop in probability as values approach zero. The joint marginal for $\rho_{s}$ and snow accretion processes PSACR, PSACI, and PSACW as well as deposition process PSDEP in Fig. 9 show a sharp rise in the activity of these processes as values of $\rho_{s}$ approach zero; additionally the activity of PSFW, the Bergeron process deposition/ riming on snow, drops precipitously. Low values of density thus strongly skew the behavior of snow accretion and deposition processes, to the extent that unrealistic profiles of reflectivity are expected. Accretion processes are related to particle cross section and fall velocity-both functions of the particle density. Indeed, as particle density approaches zero, the particle cross section will approach infinity-clearly an unrealistic result. For all these processes, one may compare the behavior at the lower extremum of $\rho_{s}$ with that displayed at this parameter's upper values-perturbations in values of snow density here produce a comparatively weaker response in snow accretion and deposition processes. Thus, the nonlinearity in parameter PDF shape is clearly shown to be related to nonlinearities in microphysical process response to parameter perturbation.

The joint parameter-process PDFs can be used to help understand the relationships displayed in the joint parameter-observation PDFs and parameter-observation covariance plots (e.g., Figs. 5 and 7). For example, in section 3 it was hypothesized that strong covariance between $N_{0 r}$ and reflectivity below the freezing line could be related to enhanced accretion processes. Indeed, upon investigation of the 2D marginals of $N_{0 \mathrm{r}}$ with QRACS and PRACW, for example, one notes strongly covariant shape. Thus, processes involving rain accretion of snow and cloud water, respectively, are enhanced when rain intercept parameter is increased-a change associated with fewer large droplets. In other cases, the parameter-process PDFs display puzzling features that do not yield readily to analysis. For example, PSFWthe Bergeron process transfer of water vapor from cloud water to snow-displays a curious trimodal structure in its marginal. These three modes are visible in most of the 2D marginals and do not seem to be associated with any clear separation in parameter value. Only in the 2D marginals between PSFW, $N_{0 \mathrm{~g}}$, and $\rho_{g}$ does there appear to be much of a relationship-a slight slope shown in the boundary between these modes.

Information concerning the parameter inversion is also provided by the joint parameter-process $2 \mathrm{D}$ marginals that do not show clear dependence. These parameterprocess relationships demonstrate a relative lack of constraint, and illustrate potential sources of uncertainty in the 
inversion of microphysical parameters. That is to say, if a process displays great variability for the full range of parameter perturbation, that process might provide compensation for other processes such that parameter constraint on the second process is nullified by compensating uncertainty from the first. For example, DGACW, graupel accretion of cloud water, shows little clear constraint from parameter perturbations, and yet it is a process that has the second highest activity during the convective phase of the storm of any process considered here.

\section{2) PARAMETER-MICROPHYSICAL PROCESS PDFS:} STRATIFORM

Few parameter-process relationships appear unchanged moving from the convective phase of the storm simulation to the stratiform phase-shown in Fig. 10. Chief among those unchanged is water vapor deposition on graupel (PGDEP) and to a lesser degree snow melting (PSMLT), snow accretion of rain (PSACR), and snow accretion of cloud water (PSACW). It is notable that two of these processes, PGDEP and PSMLT, do not involve interaction between different hydrometeor types. It has been hypothesized here that uncertainty in process dependence on parameter perturbation is partially due to complex interactions between hydrometeor types in a constantly changing storm morphology. Thus, processes with fewer interactions naturally have less uncertainty in the process-parameter relationship-a result observed here.

All other processes display differences that are in some cases substantial. For at least three processes, the relationship between parameter and process changes sign from convective to stratiform regime; these processes are evaporation of rain, ERN, graupel accretion of rain, DGACR, and rain accretion of cloud water PRACW. This result highlights a key point-that the relationship between parameter and process activity is not trivially deterministic; it is strongly dependent on model state and simulated storm morphology. In this case, all three processes showing sign change between the convective and stratiform regimes are related to rain, indicating that the role of rain depends strongly on storm morphological regime. Indeed, the activity of processes involving the accretion of rain by ice particles-PIACR, DGACR, and PSACR - are reduced by an order of magnitude in the stratiform regime relative to convective, whereas the activities of graupel melting PGMLT and rain accretion of cloud water PRACW are nearly doubled.

Another general feature of the parameter-process PDFs for the stratiform regime is the increased uncertainty in parameter-process relationship. Tightly constrained relationships between process and parameter, such as that shown between PSDEP and $\rho_{s}$ for the convective regime in Fig. 9, are conspicuously absent. This conforms to the hypothesis here linking uncertainty in microphysical processes to complex microphysical interactions. The number of possible interactions between different hydrometeors through different processes so as to produce an equivalent model state increases as the model progresses in its evolution. In addition, this points to the possibility that within the stratiform regime, model hydrometeor state provides a greater constraint on microphysical process activity than do parameters.

\section{e. Microphysical process-process PDF: Process interaction and uncertainty}

Microphysical process activity information is used to generate a 16-dimensional PDF of process activity in both convective and stratiform regions. Marginal PDFs are again shown separately for the convective (Fig. 11) and stratiform (Fig. 12) regimes of the squall-line simulation. This PDF contains the relationships between the activity of microphysical processes, both as a result of the deterministic relationships between processes as well as the stochastic relationship between processes-that is, the relationship in the uncertainties of pairs of processes. The first-order metrics for these properties are the correlations observed in the joint PDF of processes and the variances observed in the process marginals, respectively. Compared with the parameter-process PDFs presented in the previous section, the PDFs shown here more clearly display the complex interactions between hydrometeor types through a variety of microphysical processes within a varying storm environment.

More specifically, Figs. 11 and 12 help to diagnose the effect of multiple modes observed in the parameterprocess PDFs of section 3d. Considering that multiple modes observed in microphysical process activity PDFs indicate distinct regimes of microphysical behavior, the joint process-process PDF can be analyzed to determine the "trade-offs" inherent in these regimes. That is, if a process shows multiple modes of likelihood, these modes may be correlated with a similarly multimodal structure in another process, indicating which processes compensate for any increases or reductions in the activity of the first process.

Finally, the process PDFs represent the uncertainty in microphysical process activity given the chosen constraints of the inversion. The character of this uncertainty-its degree of linearity, Gaussianity, and modality-determines the ease with which it can be represented in simplified schemes. The parameter 


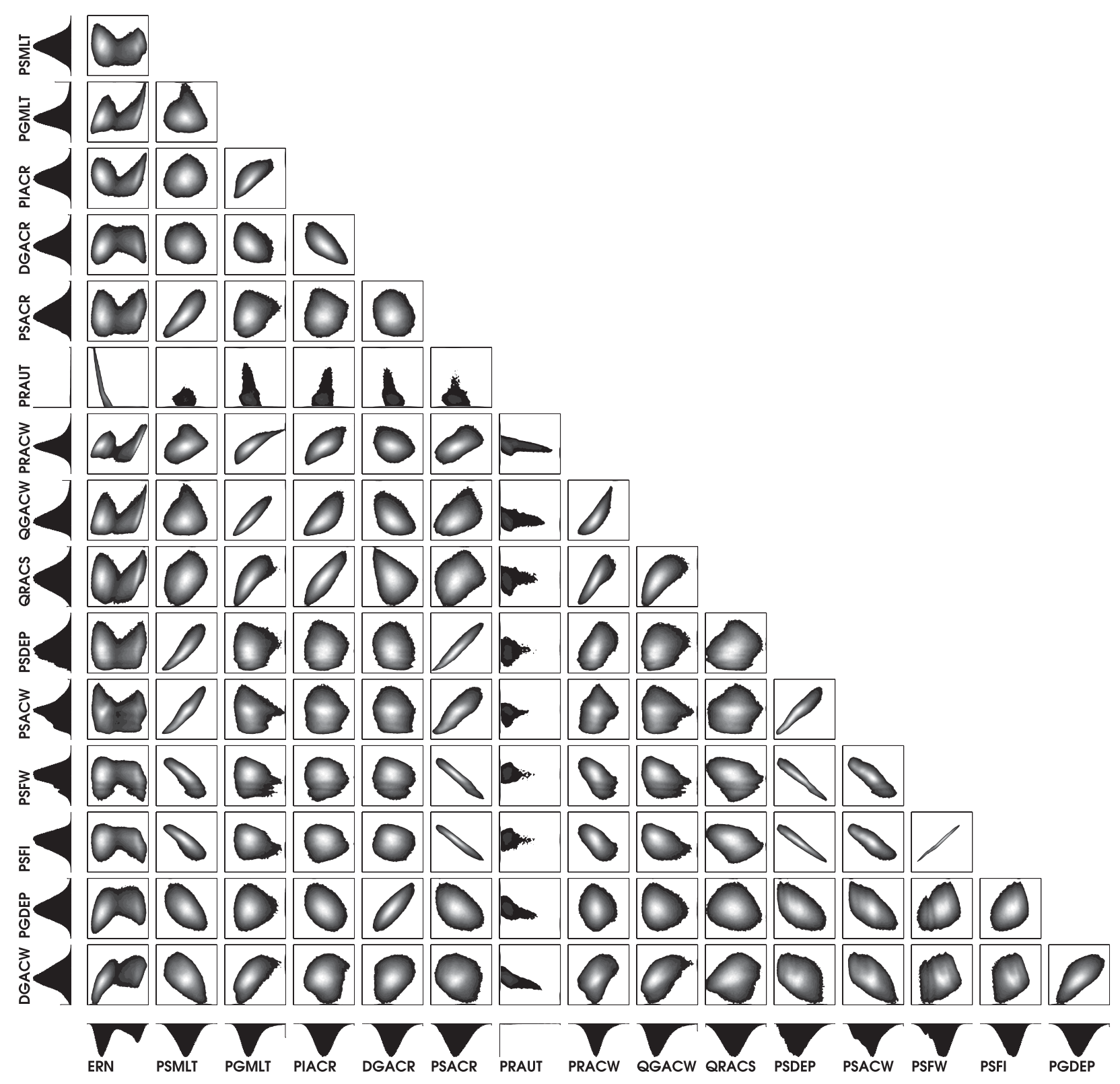

FIG. 11. Joint posterior PDFs of microphysical process activity during the convective phase of the squall-line development (50-70 min).

PDF shown in Fig. 4 displayed considerable deviations from Gaussianity in at least three parameters $\left(\rho_{s}, \rho_{g}, q_{\mathrm{c} 0}\right)$. Comparison of Figs. 11 and 12 with Fig. 4 provides insight into whether parameters or processes are better candidates to represent microphysical parameterization uncertainty within a stochastic ensemble. Furthermore, the character of the parameter information space (as indicated by the posterior PDF) gives an indication of the ability of schemes that assume a Gaussian posterior PDF (such as Kalman filters) to provide an unbiased inversion of parameters in a study using real observations (VP08).

\section{1) MicRopHysicAl PROCESS-PROCESS PDFs: CONVECTIVE REGIME}

Figure 11 displays the PDF for microphysical process activity during the convective phase of the column model integration. Here again multimodality is observed in the marginals of rain evaporation ERN and autoconversion PRAUT, associated with the bimodal structure of $q_{\mathrm{c} 0}$. Recall from the parameter-process PDF in Fig. 9 that the upper mode of $q_{\mathrm{c} 0}$ is associated with a near-zero activity in autoconversion PRAUT. It can be seen in Fig. 11 that this reduced activity is associated with a secondary mode 


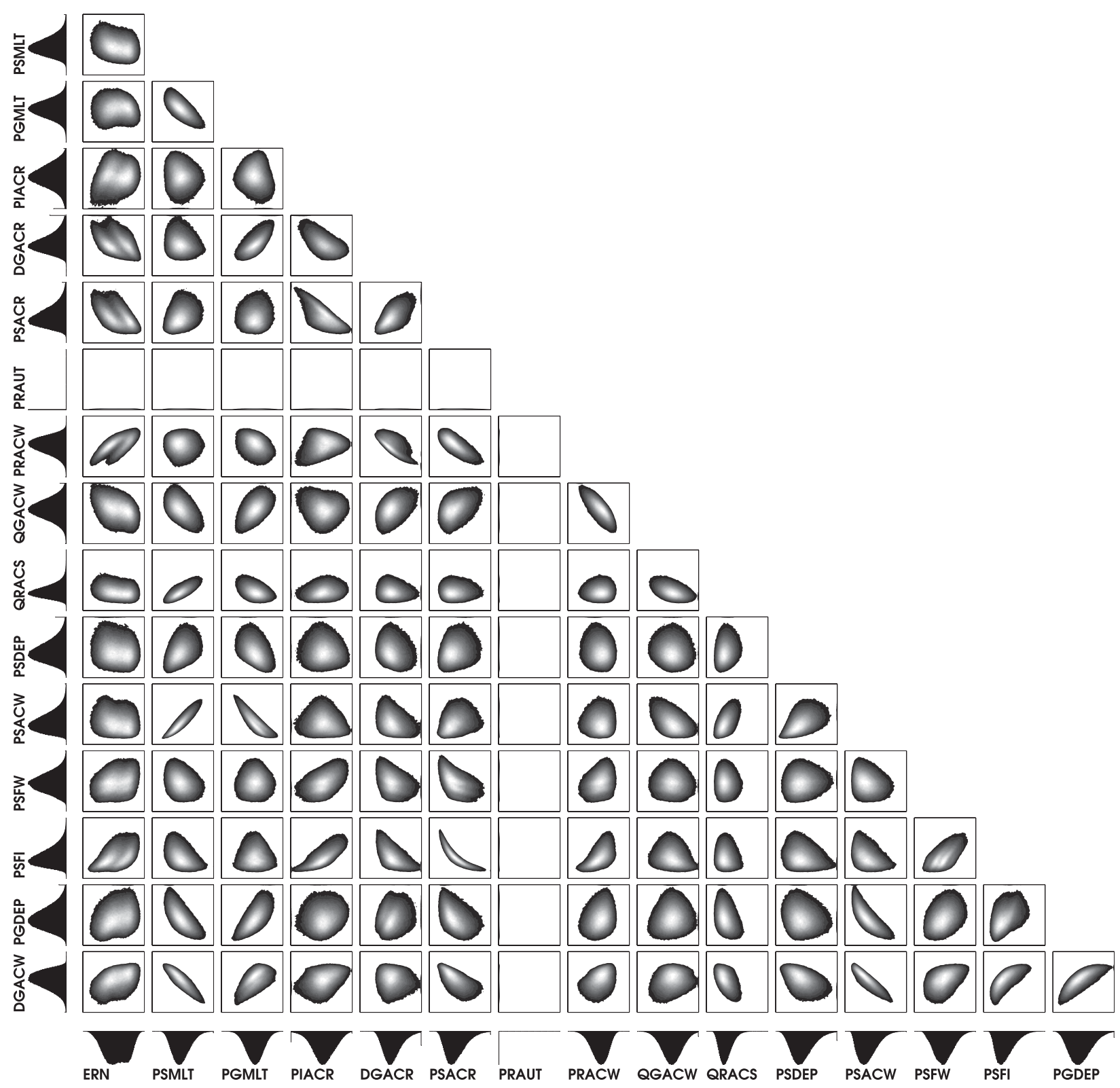

FIG. 12. As in Fig. 11, but for the stratiform phase of squall-line development (110-130 min).

in the distribution of rain evaporation ERN. Indeed, $q_{\mathrm{c} 0}$ appears to correlate well enough with ERN that conclusions drawn in section $3 \mathrm{~d}$ about the relationship between $q_{\mathrm{c} 0}$ and various microphysical processes hold here for the relationships between ERN and other processes.

The 2D marginal PDFs of process activity here serve to clarify the relationships between parameter and processes observed in Fig. 9. In the aforementioned figure, numerous processes appear to share the same deterministic relationship with a particular parameter. For example, PSACR, PSDEP, PSACW, PSFW, and PSFI all appear to maintain similarly nonlinear relationships with snow density parameter $\rho_{s}$. It is unclear from the parameter-process PDFs, however, whether this relationship is actually identical in each case. Simple inspection of process-process PDFs in Fig. 11 reveals this information; relationships between PSDEP, PSFI, PSFW, and PSACR are close to linear, while those which include PSACW are less so. In the case of PSDEP, PSACR, and PSACW, the equations controlling these processes share a dependence on $1 / \sqrt{\rho_{s}}$, however for PSFW and PSFI the deterministic dependence is less clearly specified in the equations and the process PDFs 
provide new insight (Lin et al. 1983; Rutledge and Hobbs 1983; Tao et al. 1989).

\section{2) MICROPHYSICAL PROCESS-PROCESS PDFS: STRATIFORM REGIME}

Figure 12 shows the PDF for microphysical process activity during the stratiform phase of the squall-line simulation. Generally these data display a large reduction in multimodality and nonlinearity compared with the convective regime PDFs. All 1D marginals, with the exception of ERN, are unimodal and in general, processes appear to have more uncertainty in their interrelationships. It can be seen in observational error covariance plots (Figs. 3) that the stratiform regime is expected to have greater variance in values of observed radar reflectivity - this may be seen as analogous to model error growth with time. This model error growth is visible here as a more uncertain relationship between microphysical process activities.

There are also numerous changes to the deterministic relationships of processes between the convective and stratiform regimes shown in Figs. 11 and 12. For example, the 2D marginal of PSACR and PSFI, which displayed a roughly linear relationship in Fig. 11, here displays a clearly nonlinear relationship. Other interesting examples include processes that change the sign of their first-order covariance: PRACW and QGACW. Both of these processes involve accretion of cloud water, by rain in the case of PRACW and graupel in the case of QGACW. Both processes also produce rain as the product. The positive relationship observed for the convective regime in Fig. 11 suggests that increased activity of QGACW here serves to increase rain concentrations, which in turn allows for enhanced activity of PRACW. By contrast, the negative relationship observed in the stratiform regime in Fig. 12 suggests that the limiting factor for process activity here is cloud water concentrationproduced in less abundance in the stratiform regime because of reduced updraft velocity-and that PRACW and QGACW thus compete for the accretion of cloud water. These features indicate that microphysical process uncertainty is not only correlated between processes, but also strongly dependent on storm morphology and model hydrometeor state.

A general observation can be made here about the shape of the process PDFs for both the convective and stratiform regimes. In general, the 1D marginal PDFs of microphysical processes are unimodal and symmetric, suggesting suitability for a simple Gaussian representation of process uncertainty. The $2 \mathrm{D}$ marginals display limited asymmetry and few cases of strongly curved PDFs, such as is shown in the $2 \mathrm{D}$ joint parameter marginal between $\rho_{s}$ and $N_{0 \mathrm{~s}}$ in Fig. 4. Notable exceptions include the distribution of rain evaporation, ERN, which displays multimodal structure, as well as the distribution of PSACR and PSACW, which display nonlinear 2D marginals as well as nonsymmetric $1 \mathrm{D}$ marginals.

\section{Summary and conclusions}

Uncertainty in cloud microphysical parameterization is estimated by means of a Markov chain Monte Carlo (MCMC) algorithm, which provides an inverse problem solution on 10 microphysical parameters using radar reflectivity observations with vertically covarying errors as the likelihood constraint. Radar reflectivity observations are shown to more tightly constrain microphysical parameter uncertainty than the column-integral observations used in PV10 - reducing variance and in some cases eliminating biases in the parameter inversion. In particular, ice fall speed parameters and intercept parameters of hydrometeor particle size distribution are shown to be considerably better constrained by radar reflectivity observations. Nonuniqueness shown by PV10 in the inverse solution for ice hydrometeor fall speed parameters is eliminated with the use of radar reflectivity, although the posterior PDF for cloud water-to-rain autoconversion threshold shows a bimodal structure that was not observed in PV10 for column-integral observations - a sign of nonuniqueness in the inverse solution. This property is a consequence of microphysical processes that serve to compensate for modified hydrometeor concentration associated with the spurious mode of this parameter. The bimodal structure is, in turn, a consequence of a nonmonotonic relationship between $q_{\mathrm{c} 0}$ and simulated radar reflectivity. These results demonstrate the increased information content of radar reflectivity relative to column-integral measurements as well as the utility of the probabilistic analyses employed.

Interpretation of microphysical parameter uncertainty under the constraint of radar reflectivity is facilitated by a number of novel analyses. The probabilistic approach to the solution of the inverse problem allows for the investigation of the posterior PDF of any model variable. That is, all model variables can be treated probabilistically so as to diagnose the uncertainty and sensitivity of these variables under the constraint of stochastic observations and microphysical parameter perturbation. Joint parameter-observation PDFs allow show precisely which observational levels constrain parameter uncertainty. It is found that in many cases, these relationships yield to intuitive analysis, while in other cases, sensitivity of observation to parameter perturbation is likely the product of complex microphysical interactions. For example, evaporation of rain, graupel accretion of rain, and rain accretion of cloud water are shown to change the sign of 
their first-order relationship with all parameters between the stratiform and convective storm regimes. These analyses also underscore the value of a vertically resolved observable quantity; the relationship between reflectivity and parameter perturbation is shown to be strongly height dependent.

PDFs of microphysical process activity are produced in order to further analyze the processes that contribute to the microphysical state, and thus, the observable state of the model. The results show how the behavior of microphysical processes and the relationship between uncertainty in parameters and microphysical processes is strongly dependent on storm morphology. For example, the relationship between graupel accretion of cloud water and rain accretion of cloud water changes its sign between convective and stratiform storm regimes; as is discussed in section $3 \mathrm{e}$, this is an indication that in differing storm morphological regimes, distinct microphysical processes and hydrometeor types may provide the primary constraint on microphysical behavior.

The PDF, fully in process space, allows for observation of the inter-relationships between microphysical processes. The shape of these distributions (the number of modes, skewness, and linearity of interrelationships) provides insight into the ease with which microphysical uncertainty might be represented in a parameterization scheme. Specifically, the greater the degree to which the PDF resembles a multivariate Gaussian distribution, the easier this uncertainty might be represented in forecast ensembles, and the easier it might be estimated using techniques that assume a Gaussian posterior (such as the Kalman filter). The current results show that microphysical process PDFs appear to be more symmetric and linear than parameter PDFs, with some exceptions that are noted in section 3e. This suggests that it may be more fruitful to represent microphysical parameterization uncertainty in terms of uncertain process activities rather than uncertain parameters. In the current study, however, microphysical process PDFs are limited by the fact that it is parameters, and not processes, which are directly perturbed. A future study will investigate individual microphysical processes as control parameters within a similar inverse experiment. Those results might then provide a more solid foundation for a choice of control parameters with which to best quantify microphysical parameterization uncertainty.

Acknowledgments. M. van Lier-Walqui was supported by NSF Grant AGS-1019184. D. Posselt was supported by NASA Modeling, Analysis and Prediction Grants NNX09AJ43G and NNX09AJ46G, as well as an Office of Naval Research Grant N00173-10-1-G035.

\section{REFERENCES}

Clark, A. J., W. A. Gallus Jr., and T.-C. Chen, 2008: Contributions of mixed physics versus perturbed initial/lateral boundary conditions to ensemble-based precipitation forecast skill. Mon. Wea. Rev., 136, 2140-2156.

Dearden, C., P. J. Connolly, T. W. Choularton, and P. R. Field, 2010: Evaluating the effects of microphysical complexity in idealised simulations of trade wind cumulus using the Factorial Method. Atmos. Chem. Phys. Discuss., 10, 23 497-23 537, doi:10.5194/acpd-10-23497-2010.

Gallus, W. A., Jr., and M. Pfeifer, 2008: Intercomparison of simulations using 5 WRF microphysical schemes with dualpolarization data for a German squall line. Adv. Geosci., 16, 109-116.

Golaz, J.-C., V. E. Larson, J. A. Hansen, D. P. Schanen, and B. M. Griffin, 2007: Elucidating model inadequacies in a cloud parameterization by use of an ensemble-based calibration framework. Mon. Wea. Rev., 135, 4077-4096.

Haynes, J. M., R. T. Marchand, Z. Luo, A. Bodas-Salcedo, and G. L. Stephens, 2007: A multipurpose radar simulator package: QuickBeam. Bull. Amer. Meteor. Soc., 88, 1723-1727.

Jackson, C., Y. Xia, M. K. Sen, and P. L. Stoffa, 2003: Optimal parameter and uncertainty estimation of a land surface model: A case study using data from Cabauw, Netherlands. J. Geophys. Res., 108, 4583, doi:10.1029/2002JD002991.

, M. K. Sen, G. Huerta, Y. Deng, and K. P. Bowman, 2008: Error reduction and convergence in climate prediction. J. Climate, 21, 6698-6709.

Jankov, I., W. A. Gallus Jr., M. Segal, and S. E. Koch, 2007: Influence of initial conditions on the WRF-ARW model QPF response to physical parameterization changes. Wea. Forecasting, 22, 501-519.

Järvinen, H., P. Räisänen, M. Laine, J. Tamminen, A. Ilin, E. Oja, A. Solonen, and H. Haario, 2010: Estimation of ECHAM5 climate model closure parameters with adaptive MCMC. Atmos. Chem. Phys., 10, 9993-10 002.

_- M. Laine, A. Solonen, and H. Haario, 2012: Ensemble prediction and parameter estimation system: The concept. Quart. J. Roy. Meteor. Soc., 138, 281-288, doi:10.1002/ qj. 923.

Lang, S., W.-K. Tao, R. Cifelli, W. Olson, J. Halverson, S. Rutledge, and J. Simpson, 2007: Improving simulations of convective systems from TRMM LBA: Easterly and westerly regimes. J. Atmos. Sci., 64, 1141-1164.

Li, X., W.-K. Tao, A. P. Khain, J. Simpson, and D. E. Johnson, 2009: Sensitivity of a cloud-resolving model to bulk and explicit bin microphysical schemes. Part I: Comparisons. J. Atmos. Sci., 66, 3-21.

Lin, Y.-L., R. D. Farley, and H. D. Orville, 1983: Bulk parameterization of the snow field in a cloud model. J. Climate Appl. Meteor., 22, 1065-1092.

Liu, Y., H. V. Gupta, S. Sorooshian, L. A. Bastidas, and W. J. Shuttleworth, 2004: Exploring parameter sensitivities of the land surface using a locally coupled land atmosphere model. J. Geophys. Res., 109, D21101, doi:10.1029/2004JD004730.

Masunaga, H., and Coauthors, 2010: Satellite data simulator unit: A multisensor, multispectral satellite simulator package. Bull. Amer. Meteor. Soc., 91, 1625-1632.

McCumber, M., W.-K. Tao, J. Simpson, R. Penc, and S.-T. Soong, 1991: Comparison of ice-phase microphysical parameterization schemes using numerical simulations of tropical convection. J. Appl. Meteor., 30, 985-1004. 
Metropolis, N., A. W. Rosenbluth, M. N. Rosenbluth, A. H. Teller, and E. Teller, 1953: Equation of state calculations by fast computing machines. J. Chem. Phys., 21 (6), 1087-1092.

Milbrandt, J. A., and M. K. Yau, 2005: A multimoment bulk microphysics parameterization. Part I: Analysis of the role of the spectral shape parameter. J. Atmos. Sci., 62, 3051-3064.

Morrison, H., and W. W. Grabowski, 2007: Comparison of bulk and bin warm-rain microphysics models using a kinematic framework. J. Atmos. Sci., 64, 2839-2861.

—, G. Thompson, and V. Tatarskii, 2009: Impact of cloud microphysics on the development of trailing stratiform precipitation in a simulated squall line: Comparison of one- and two-moment schemes. Mon. Wea. Rev., 137, 991-1007.

Mosegaard, K., and A. Tarantola, 2002: Probabilistic approach to inverse problems. International Handbook of Earthquake \& Engineering Seismology, W. H. K. Lee et al., Eds., Academic Press, 237-265.

Ott, L. E., and Coauthors, 2009: Analysis of convective transport and parameter sensitivity in a single column version of the Goddard Earth Observation System, version 5, general circulation model. J. Atmos. Sci., 66, 627-646.

Posselt, D. J., 2006: Application of advanced data assimilation techniques to the study of cloud and precipitation feedbacks in the tropical climate system. Ph.D. thesis, Colorado State University, Fort Collins, CO, 277 pp.

—_, and T. Vukicevic, 2010: Robust characterization of model physics uncertainty for simulations of deep moist convection. Mon. Wea. Rev., 138, 1513-1535.

— T. T. L'Ecuyer, and G. L. Stephens, 2008: Exploring the error characteristics of thin ice cloud property retrievals using a Markov chain Monte Carlo algorithm. J. Geophys. Res., 113, D24206, doi:10.1029/2008JD010832.

Robert, C., and G. Casella, 2004: Monte Carlo Statistical Methods. 2nd ed. Springer Verlag, 645 pp.

Rutledge, S. A., and P. V. Hobbs, 1983: The mesoscale and microscale structure and organization of clouds and precipitation in midlatitude cyclones. Part VIII: A model for the "seederfeeder" process in warm-frontal rainbands. J. Atmos. Sci., 40, 1185-1206.

Sen, M. K., and P. L. Stoffa, 1996: Bayesian inference, Gibbs' sampler and uncertainty estimation in geophysical inversion. Geophys. Prospect., 44, 313-350.

Shipway, B. J., and A. A. Hill, 2012: Diagnosis of systematic differences between multiple parametrizations of warm rain microphysics using a kinematic framework. Quart. J. Roy. Meteor. Soc., doi: 10.1002/qj.1913, in press.

Stensrud, D. J., J.-W. Bao, and T. T. Warner, 2000: Using initial condition and model physics perturbations in short-range ensemble simulations of mesoscale convective systems. Mon. Wea. Rev., 128, 2077-2107.

Tao, W.-K., and J. Simpson, 1993: Goddard Cumulus Ensemble Model. Part I: Model description. Terr. Atmos. Oceanic Sci., 4 (1), 35-72.

$\longrightarrow, \ldots$, and M. McCumber, 1989: An ice-water saturation adjustment. Mon. Wea. Rev., 117, 231-235.

_ , and Coauthors, 2003: Microphyics, radiation and surface processes in the Goddard Cumulus Ensemble (GCE) model. Meteor. Atmos. Phys., 82, 97-137.

Tarantola, A., 2005: Inverse Problem Theory and Methods for Model Parameter Estimation. SIAM, 352 pp.

Thompson, G., R. M. Rasmussen, and K. Manning, 2004: Explicit forecasts of winter precipitation using an improved bulk microphysics scheme. Part I: Description and sensitivity analysis. Mon. Wea. Rev., 132, 519-542.

Tong, M., and M. Xue, 2008: Simultaneous estimation of microphysical parameters and atmospheric state with simulated radar data and ensemble square root Kalman filter. Part I: Sensitivity analysis and parameter identifiability. Mon. Wea. Rev., 136, 1630-1648.

Vukicevic, T., and D. Posselt, 2008: Analysis of the impact of model nonlinearities in inverse problem solving. J. Atmos. Sci., 65, 2803-2823. 